\title{
NRN1 and CAT Gene Polymorphisms, Complex Noise, and Lifestyles Interactively Affect the Risk of Noise-Induced Hearing Loss
}

\section{Shuang-yan LIU}

Hangzhou Normal University https://orcid.org/0000-0002-9261-2025

Jia-Rui XIN

Hangzhou Normal University

\section{Zheng LI}

Hangzhou Normal University

\section{Song LEI}

Ningbo center for disease control and prevention

\section{Ying-Qi CHEN}

Hangzhou Normal University

\section{Tian-Yu ZHAO}

Central people' hospital of zhanjiang

\section{Hai-Yan WANG}

Hangzhou Normal University

\section{Liang-Wen XU}

Hangzhou Normal University

\section{Mei-Bian ZHANG}

Zhejiang Provincial CDC: Zhejiang Provincial Center for Disease Control and Prevention

Lei YANG ( $\sim$ yanglei62@hznu.edu.cn )

Hangzhou Normal University https://orcid.org/0000-0001-7618-9936

\section{Research}

Keywords: noise-induced hearing loss, kurtosis, CAT, NRN1, lifestyle, interaction, generalized multifactor dimensionality reduction

Posted Date: October 19th, 2020

DOI: https://doi.org/10.21203/rs.3.rs-93355/v1

License: (c) (i) This work is licensed under a Creative Commons Attribution 4.0 International License. 

NRN1 and CAT gene polymorphisms, complex noise, and lifestyles interactively affect the risk of noise-induced hearing loss

Shuang-Yan LIU ${ }^{1+}$, Jia-Rui XIN ${ }^{1+}$, Zheng LI ${ }^{1}$, Song LEI ${ }^{2}$, Ying-Qi CHEN ${ }^{1}$, Tian-Yu ZHAO ${ }^{3}$, Hai-Yan WANG ${ }^{1}$, Liang-Wen XU ${ }^{1}$, Mei-Bian ZHANG ${ }^{4 *}$, Lei YANG ${ }^{1^{*}}$

1 Medical School, Hangzhou Normal University, Hangzhou 310000, China

2 Ningbo Center for Disease Control and Prevention, Ningbo 315700, China

3 Central people`s hospital of Zhanjiang, Zhanjiang 524000, China

4 Institute of Occupational Health and Radiation Protection, Zhejiang Provincial Center for Disease

Control and Prevention, Hangzhou 310000, China

\section{*Correspondence:}

Dr. Mei-Bian ZHANG

Zhejiang Provincial Center for Disease Control and Prevention, Hangzhou, China

E-mail: mbzhang@cdc.zj.cn

Professor Lei YANG, PhD

Hangzhou Normal University, Zhejiang, China

Tel: $+86-571-28865010$

Fax: $+86-571-28865000$

E-mail: yanglei62@hznu.edu.cn

${ }^{+}$These authors contributed equally to this work.

\section{(1)}




\section{ABSTRACT}

Background: Multiple genetic and environmental factors influence the severity of NIHL. However, few studies have reported interactions among such factors in modulating the risk of NIHL. This study aimed to assess for interactions among gene polymorphisms, noise metrics, and lifestyles on the risk of NIHL.

Methods: A case-control study was conducted using 307 patients with NIHL and 307 matched healthy individuals from five manufacturing industries. General demographic data, lifestyle details, and noise exposure levels were recorded. The kompetitive allele-specific polymerase chain reaction (KASP) was used to analyze the genotypes of 18 single nucleotide polymorphisms (SNPs). The generalized multifactor dimensionality reduction (GMDR) method was used to examine the effects of all possible interactions.

Results: The proportion of people with complex noise exposure, high CNE, high adj-CNE, smoking, propensity to watch loud videos, or sedentary lifestyle was significantly greater in the NIHL group than in the healthy group $(P<0.05)$. The GMDR model demonstrated a relevant interaction between NRN1 rs3805789 and CAT rs7943316. Subjects with the SNP pair of NRN1 rs3805789-CC and CAT rs7943316-AT, NRN1 rs3805789-CT and CAT rs7943316-AA, NRN1 rs3805789-CT and CAT rs7943316-TT, NRN1 rs3805789-CT/TT and CAT rs7943316-AA, or NRN1 rs3805789-CC and CAT rs7943316-AT/TT had higher risks of NIHL than those with NRN1 rs3805789-CC and CAT rs7943316-AA $(P<0.05)$. There was an interaction among NRN1 rs3805789, CAT rs7943316, and kurtosis. Subjects exposed to complex noise and carrying both NRN1 rs3805789-CT and CAT rs7943316-TT or NRN1 rs3805789-CT/TT and CAT rs7943316-AA had higher risks of NIHL than those exposed to steady noise and carrying both NRN1 rs3805789-CC and CAT rs7943316-AA ( $P$ 
$<0.05$ ). The best six-locus model involving NRN1 rs3805789, CAT rs7943316, smoking, video volume, physical exercise, and working pressure for the risk of NIHL was found to be the interaction $(P=0.0010)$. An interaction was also found among smoking, video volume, physical exercise, working pressure, and kurtosis $(P=0.0107)$.

Conclusions: Complex noise, high $\mathrm{CNE}$, high adj-CNE, smoking, high video volume, and sedentary lifestyle are environmental risk factors for NIHL. Concurrence of NRN1 rs3805789 and CAT rs7943316 constitutes a genetic risk factor for NIHL. Complex noise exposure significantly increases the risk of NIHL in subjects with a high genetic risk score. Interactions between genes and lifestyle as well as noise metrics and lifestyle affect the risk of NIHL. These results provide a theoretical basis for screening genetic and environmental risk factors to prevent NIHL.

Keywords: noise-induced hearing loss; kurtosis; CAT; NRN1; lifestyle; interaction; generalized multifactor dimensionality reduction

\section{Introduction}

Noise-induced hearing loss (NIHL) is a slowly progressive sensorineural hearing loss caused by long-term exposure to harmful levels of noise. The World Health Organization (WHO) estimates that approximately $22 \%$ of the hearing loss in adults is attributable to occupational and environmental noise exposure, and by 2030, almost 1 billion people will be at the risk of NIHL[1] .

As a major occupational health risk, NIHL has become the second-largest occupational disease in China [2]. NIHL is thought to be a complex disease caused by genetic and environmental factors. The main factors include exposure to high levels of noise and individual susceptibility, such as age, gender, education level, smoking frequency, alcohol consumption, and usage practice of hearing 
protection devices [3-6]. Therefore, the single-locus method may not be appropriate to study common complex disorders such as NIHL.

Noise is the most common environmental factor leading to occupational hearing loss. The noise exposure metrics used in most previously published studies mainly concentrates on equivalent continuous sound level $\left(\mathrm{L}_{\mathrm{eq}}\right)$ and cumulative noise exposure $(\mathrm{CNE})$. These metrics have been established based on the study of Gaussian noise and the equal-energy hypothesis (EEH), which assumes that the damage to the auditory system caused by noise exposure is proportional to the duration of exposure multiplied by the noise intensity. However, the EEH has been found unsuitable for "complex noise or non-Gaussian (non-G)" noise. Complex noise is ubiquitous in industrial and military environments. It is composed of a transient high-energy impulsive noise superimposed on stationary (Gaussian) background noise [7]. Both animal experiments and epidemiological studies have shown that the EEH underestimates the cochlear impact of complex-noise exposure. The impact of a complex-noise-induced impulse on the auditory system was assessed using kurtosis first by Erdreich [8]. This method has simplified the time-domain variables of noise that affect hearing (e.g., pulse peak value, duration, and inter-pulse distribution) into one easy-to-calculate parameter (i.e., kurtosis), which is convenient for classifying the noise type. A high kurtosis indicates that the impulse of the complex noise was high [9]. To date, the efficacy of kurtosis in assessing complex noise has been preliminarily verified in human studies $[10,11]$.

Increasing evidence has shown the association of susceptibility genes, such as catalase (CAT), heat shock protein70 (HSP70), cadherin-23 (CDH23), caspase (CASP), and NADPH oxidase3 (NOX3), with the development of NIHL [12-18]. Additionally, previous studies have demonstrated that smoking, stressful lifestyle, and physical exercise are associated with hearing loss [19-21]. 
However, few studies have analyzed the interaction between genetic variants, noise exposure (especially kurtosis), and lifestyle factors on modulating NIHL. Previous studies have never reported multidimensional interactions involving multiple $(>7)$ genes [especially the Neuritin1 (NRN1) gene] and kurtosis. Therefore, in a case-control study with 307 NIHL patients and 307 ageand gender-matched healthy controls, a total of 18 variants in these 7 susceptibility genes $(C A T$, HSP70, CDH23, CASP3, CASP7, NOX3, and NRN1), three noise metrics (noise kurtosis, CNE, adj$\mathrm{CNE}$ ), and four lifestyle factors (smoking, video volume, physical exercise, working pressure) were included to explore the associations of gene-gene, gene-noise-metric, gene-lifestyle-factor, and noise-metric-lifestyle-factor interactions with the risk of NIHL. Our results lay the foundation for a comprehensive prevention program against NIHL.

\section{Methods}

\section{Subjects}

Subjects were continuously recruited between October 2017 and December 2018 from five manufacturing factories with high noise levels in the Zhejiang Province of East China. Inclusion criteria for the subjects were as follows: (1) individuals who had never worked in high noise-level environments from different enterprises, (2) the binaural hearing threshold difference was $<30 \mathrm{~dB}$ per frequency; (3) no history of military service; (4) no family history of hearing loss; (5) no history of an ear disease; (6) no history of ototoxic drugs; and (7) no history of diabetes. NIHL was diagnosed based on binaural high-frequency $(3000,4000$, and $6000 \mathrm{~Hz})$ average hearing threshold $>$ $25 \mathrm{~dB}$. The subjects were divided into two groups - patients with NIHL $(\mathrm{n}=307)$ and controls with normal hearing $(\mathrm{n}=307)$ —who were matched for gender and age $( \pm 3$ years $)$. 


\section{Questionnaire survey}

A questionnaire was designed for each subject based on the needs of the investigation. Collected information included the following: (1) general information (age, sex, etc.); (2) noise exposure factors (factory, work situation, duration of daily noise exposure, etc.); (3) lifestyle factors (smoking, video volume, physical exercise and working pressure). Variables were defined as follows: (1) smoking: average daily cigarette use for $\geq 1$ year; (2) video volume: high video volume $\geq 40 \%$ of the maximum volume; low video volume $<40 \%$ of the maximum volume; (3) regular physical exercise: average physical exercise for once a month or more often for $\geq 1$ year. Participants completed study questionnaires and met with trained investigators in a face-to-face interview. All the participants signed the informed consent form, and the study was approved by the Science Ethics Committee of Hangzhou Normal University (2017LL107).

\section{Noise waveform recording and analysis}

A digital noise dosimeter (ASV5910-R, Hangzhou Aihua Instrument Co., Ltd.) that can operate continuously at a sampling rate of $48 \mathrm{kHz}$ was used to record the noise for each subject for the entire shift duration. Eight-hour, continuous equivalent A-weighted sound levels $\left(\mathrm{L}_{\text {Aeq,8h }}\right)$ can be measured with a noise dosimeter, which was attached to the clothing of the participant at the shoulder by clips, with the microphone pointed up (Supplementary material 1). The measurement time was 8 hours per shift. A sound level calibrator (Hangzhou Aihua Instrument, AWA6221B) was used to calibrate the noise dosimeter before and after each sampling cycle. MATLAB software (Natick, MA) was used to calculate the sampling kurtosis in a continuous 40 -s window of the noise signals during the entire shift. The equation used to calculate kurtosis is shown in Formula 1.

$$
\beta=\frac{\mathrm{m}_{4}}{\mathrm{~m}_{2}}=\frac{\frac{1}{\mathrm{n}} \sum_{\mathrm{i}=1}^{\mathrm{n}}\left(\mathrm{x}_{\mathrm{i}}-\overline{\mathrm{x}}\right)^{4}}{\left(\frac{1}{\mathrm{n}} \sum_{\mathrm{i}=1}^{\mathrm{n}}\left(\mathrm{x}_{\mathrm{i}}-\overline{\mathrm{x}}\right)^{2}\right)^{2}}
$$


where, $\mathrm{x}_{\mathrm{i}}$ is the $\mathrm{i}^{\text {th }}$ value, $\bar{x}$ is the sample mean, and $\beta$ is noise kurtosis. Theoretically, the kurtosis value of Gaussian noise is $3(\beta=3)$ and that of complex non-Gaussian noise is greater than 3 . The larger the kurtosis value, the higher the impulse of the complex noise. The selection of a 40-s window is acceptable for kurtosis measurement based on a 48-kHz sampling rate, as observed from previous animal data $[22,23]$. The median kurtosis calculated in a 40-s window was used as the kurtosis value of the entire shift time. In this study, a median kurtosis of 4 was used as the boundary value between Gaussian and complex non-Gaussian noise.

Both noise level and noise exposure time should be used to assess NIHL. Therefore, a comprehensive noise exposure metric (CNE) was used to quantify noise energy for each worker according to Formula 2 [10]:

$$
\mathrm{CNE}=\mathrm{L}_{\mathrm{Aeq}, 8 \mathrm{~h}}+10 \log \mathrm{T}
$$

where, $\mathrm{L}_{\mathrm{Aeq}, 8 \mathrm{~h}}$ is the equivalent continuous A-weighted noise exposure level normalized to an 8 -h working day and $\mathrm{T}$ is the time of noise exposure in years. CNE is measured in $\mathrm{dB}(\mathrm{A})$ per year.

To incorporate kurtosis $(\beta)$ into the evaluation of complex noise environments and unify CNE calculations for epidemiologic data, including both Gaussian and complex noise, the kurtosisadjusted CNE (adj-CNE) was calculated according to Formula 3 [10]:

$$
\text { adj }-\mathrm{CNE}_{\text {Kurtosis-adjusted }}=\mathrm{L}_{\mathrm{Aeq}, 8 \mathrm{~h}}+\frac{\ln (\beta)+1.9}{\log (2)} \log \mathrm{T}
$$

When Gaussian noise has a kurtosis of $\beta=3$, the term $\left[\frac{\ln (\beta)+1.9}{\log (2)}\right]$ becomes equal to 10 . Thus, for Gaussian noise, the adj-CNE equals the unadjusted CNE. Equation (3) shows that when $\mathrm{L}_{\mathrm{Aeq}, 8 \mathrm{~h}}$ is fixed, the adj-CNE will be larger for complex noise $(\beta>3)$ than that for Gaussian noise $(\beta=3)$.

\section{Hearing testing and hearing loss diagnosis}

Experienced otolaryngologists performed pure-tone audiometry for the left and right ears of 
each participant at 500,1000, 2000,3000,4000, 6000, and $8000 \mathrm{~Hz}$ in a sound-insulated room with background noise $<25 \mathrm{~dB}$ (A) (Supplementary material 2). All the subjects were required to be outside of their daily noise environment for at least $16 \mathrm{~h}$ before the test. The results of the pure-tone audiometry were adjusted according to gender and age by following the ISO 1999-2013 standard. High-frequency NIHL (hNIHL) was diagnosed based on binaural high-frequency hearing threshold levels at 3, 4, and $6 \mathrm{kHz}\left(\mathrm{HTL}_{3,4,6)}\right)$ using Formula 4:

$$
\mathrm{HTL}_{346}=\frac{\text { Left }\left(\mathrm{HL}_{3 \mathrm{KHz}}+\mathrm{HL}_{4 \mathrm{KHz}}+\mathrm{HL}_{6 \mathrm{KHz}}\right)+\mathrm{Right}\left(\mathrm{HL}_{3 \mathrm{KHz}}+\mathrm{HL}_{4 \mathrm{KHz}}+\mathrm{HL}_{6 \mathrm{KHz}}\right)}{6}
$$

A binaural threshold $>25 \mathrm{~dB}$ was considered binaural hNIHL [16].

\section{Genomic DNA extraction, single nucleotide polymorphism (SNP) selection, and genotyping}

Oral mucosa cells from all the participants were collected using Yongming flocking swabs.

DNA was extracted using the Tiangen Oral Mucosa Genomic DNA extraction kit (Tiangen Biotech, Beijing, China). For SNP analysis, 18 SNPs were selected from 7 genes (CAT, HSP70, CDH23, CASP3, CASP7, NOX3, and NRN1). The SNP selection process has previously been described [17]. The detailed information about the screened SNPs is shown in Table 1. We performed the genotyping analysis using the Kompetitive allele-specific polymerase chain reaction (KASP) method as previously described [17]. The primer and probe sequences are shown in Tables S1 and S2. To control the quality, we randomly selected $10 \%$ of the samples and re-classified the genes; the concordance of the 18 SNPs was $>95 \%$.

\section{Statistical analysis}

Normally distributed continuous variables are expressed as mean \pm standard deviation (SD), and categorical variables are presented as percentages. Student's $t$-test and the Chi-square test were used to compare the continuous variables and categorical variables, respectively, between the cases 
and controls. Non-normally distributed continuous variables were expressed as median (with the lower and upper quartiles) $[\mathrm{M}(\mathrm{P} 25, \mathrm{P} 75)]$ and analyzed using the Mann-Whitney U test. The cutoff values for the CNE and adj-CNE were determined to be $97.1420 \mathrm{~dB}(\mathrm{~A})$ and $96.9939 \mathrm{~dB}(\mathrm{~A})$, respectively, based on the receiver operating characteristic (ROC) curve between CNE, adj-CNE, and NIHL. The Hardy-Weinberg equilibrium (HWE) was tested using the Chi-square test. The generalized multifactor dimensionality reduction (GMDR Software Beta 0.9, www.ssg.uab.edu/gmdr/) method [24] was used to examine the effects of all possible interactions. The sign test of cross-validation consistency (CVC), testing balanced accuracy (TEBA), and trained balanced accuracy (TRBA) were calculated. A multivariate logistic regression model was used for the stratified analysis of the significant interactions obtained from the GMDR. Multiple comparisons were corrected using the Benjamini-Hochberg procedure. $P<0.05$ indicated that the differences were statistically significant (shown in bold in the following tables).

\section{Results}

A total of 614 participants (474 males and 140 females), including 307 NIHL patients and 307 controls, were selected (Table 2). The median age of the subjects was 35 years. The median kurtosis in the NIHL group was 7.25 (4.63-14.30), which was significantly higher than that in the control group [5.85 (4.06-12.51); $P=0.006]$. The proportion of the subjects exposed to complex noise $(\beta$ $\geq 4)$ was significantly greater in the NIHL group than that in the control group $(P=0.038)$. The median HTL $_{346}$ in the NIHL group was $36.83(29.83-49.83) \mathrm{dB}$, which was significantly higher than that in the control group $[17.83(14.17-21.00) \mathrm{dB} ; P<0.001)$. The proportion of the subjects with high CNE ( $\geq 97.1420)$, high adj-CNE ( $\geq 96.9939)$, smoking habit, propensity to watch videos at 
high volume, or sedentary lifestyle was significantly greater in the NIHL group than in the control group $(P<0.05)$. However, there was no significant difference in education level or working pressure between the two groups $(P>0.05)$.

The genotype frequencies among the cases and controls did not deviate from the HWE for any of the 18 SNPs $(P>0.05$, Table 3). We assessed for NIHL-related interactions among the 18 genetic variants by using GMDR. Consequently, 18 models were generated from the 18 SNPs (Table 4). After adjusting age, gender, education level, years of noise exposure, kurtosis, CNE, adj-CNE, smoking, video volume, physical exercise, and working pressure, a significant two-locus model $(P$ $=0.0107$ ) involving NRN1 rs3805789 and CAT rs7943316 was found (Table 4, Figure S1). The CVC of this two-locus model was 10/10, and the TEBA was 0.5768 . We then conducted a stratified analysis for the significant models by using logistic regression. When compared with the subjects carrying NRN1 rs3805789-CC and CAT rs7943316-AA, those with NRN1 rs3805789-CC and CAT rs7943316-AT, NRN1 rs3805789-CT and CAT rs7943316-AA, NRN1 rs3805789-CT and CAT rs7943316-TT, NRN1 rs3805789-CT/TT and CAT rs7943316-AA, or NRN1 rs3805789-CC and CAT rs7943316-AT/TT had higher risks of NIHL (OR: 2.276, 95\% CI: 1.171-4.427; OR: 2.213, 95\% CI: 1.273-3.849; OR: 3.169, 95\% CI: 1.425-7.048; OR: 2.005, 95\% CI: 1.200-3.348; OR: 1.892 , 95\% CI: 1.008-3.550; $P<0.05)$ (Figure 1, Table S3).

We next asked whether there were any multidimensional interactions between the genes and noise metrics by using the GMDR method. After adjustments were made for age, gender, education, smoking, video volume, physical exercise, and working pressure, the best model for the risk of NIHL was found to be the interaction between NRN1 rs3805789, CAT rs7943316, and kurtosis. This interaction had the score of $10 / 10$ for $C V C$ and 10 for the sign test $(P=0.0010$; Table 5A, Figure 
S2). The joint effects of the individual interactions of NRN1 rs3805789 and CAT rs7943316 with kurtosis on NIHL risk were analyzed via logistic regression analysis. The results showed that, after adjusting age, gender, education level, years of noise exposure, smoking, video volume, physical exercise, and working pressure, the subjects exposed to complex noise who carried NRNI rs3805789-CT and CAT rs7943316-TT or NRN1 rs3805789-CT/TT and CAT rs7943316-AA had higher risks of NIHL than those exposed to steady noise who carried NRN1 rs3805789-CC and CAT rs7943316-AA (OR: 5.961, 95\% CI: 1.219-29.155; OR: 1.607, 95\% CI: 1.035-2.494; $P<0.05$ )

(Figure 2, Table S4). In the GMDR model, a two-locus model including NRN1 rs3805789 and CAT rs7943316 was found to be significant. This observation is consistent with the results of gene-gene interactions. A four-locus model including NRN1 rs3805789, CAT rs7943316, kurtosis, and adjCNE was found to be the interaction, in which the CVC was 10/10, and the TEBA was 0.5856 $(P=0.0107$; Table 5A $)$. In addition, a five-locus model was also identified for the risk of NIHL. In this model, the CVC was $10 / 10$, and TEBA was $0.5856(P=0.0107$; Table 5A).

The GMDR model was used to screen for the best gene-lifestyle-factor combinations. After adjusting age, gender, education level, years of noise exposure, kurtosis, CNE, and adj-CNE, the best six-locus model involving NRN1 rs3805789, CAT rs7943316, smoking, video volume, physical exercise, and working pressure for the risk of NIHL was found to be the interaction, which scored 10/10 for CVC and 9 for the sign test $(P=0.0010$; Table 5B). A four-locus model involving NRNI rs3805789, CAT rs7943316, smoking, and physical exercise was found to be the interaction, which scored $9 / 10$ for CVC and 9 for sign test $(P=0.0107$; Table 5B). A three-locus model involving NRN1 rs3805789, CAT rs7943316, and working pressure was found to be the interaction, which scored 5/10 for CVC and 9 for the sign test $(P=0.0107$; Table 5B). Moreover, a five-locus model 
was also identified for the risk of NIHL. The corresponding CVC and TEBA were 7/10 and 0.5570, respectively $(P=0.0107$; Table 5B $)$.

We next evaluated the interaction combinations between noise metrics and lifestyle factors via the GMDR model. The results revealed that, after adjusting age, gender, and education level, a fivelocus model involving smoking habit, video volume, physical exercise, working pressure, and kurtosis was found to be the interaction, which scored $10 / 10$ for CVC and 9 for the sign test $(P=0.0107$; Table 5C). A four-locus model involving smoking, video volume, physical exercise, and working pressure was found to be the interaction $(P=0.0107$; Table 5C), in which the CVC was 10/10, and the TEBA was 0.5509. A seven-locus model involving smoking, video volume, physical exercise, working pressure, kurtosis, CNE, and adj-CNE was found to be the interaction, which scored 10/10 for CVC and 9 for the sign test $(P=0.0107$; Table 5C). Furthermore, a sixand 0.5437 , respectively $(P=0.0107$; Table 5C).

\section{Discussion} adjusting confounding covariates, but also explores complex multi-locus interactions between 
applied to analyze the associations of gene-gene and gene-environment interactions with many complex diseases [25-27].

Increasing evidence has shown that multiple genes are closely associated with susceptibility to NIHL. Given that multiple genetic loci with moderate effects fail to reach genome-wide significance due to the limited power in most genetic studies [28], the present study focused on the associations of multi-locus interactions with NIHL risk by analyzing 18 variants in 7 susceptibility genes via the GMDR method. These genes were CAT, HSP70, CDH23, CASP3, CASP7, NOX3, and NRN1. These risk genes play significant roles in apoptosis, cell adhesion, and oxidative stress during the development of NIHL. We identified for the first time that the interaction between NRN1 rs3805789 and $C A T$ rs7943316 increased susceptibility to NIHL. We further validated this genetic interaction via stratified analysis. The results illustrated that subjects carrying NRN1 rs3805789-CC and CAT rs7943316-AT, NRN1 rs3805789-CT and CAT rs7943316-AA, NRN1 rs3805789-CT and CAT rs7943316-TT, NRN1 rs3805789-CT/TT and CAT rs7943316-AA, or NRN1 rs3805789-CC and CAT rs7943316-AT/TT had higher risks of NIHL than those with NRN1 rs3805789-CC and CAT rs7943316-AA. Yang et al. [13] found that CAT rs208679 and rs769217 were significantly associated with the risk of NIHL. A study by Wang et al. [12] have studied the association of CAT rs7943316 with NIHL susceptibility. Their results indicated that carriers of T allele (AT+TT) of rs7943316 have significantly higher risks of NIHL than those with AA genotype $(P<0.05)$, and observed that a significant interaction model involving GJB2 rs4880, SOD2 rs137852540, and CAT rs769214 might associated with NIHL. These results are similar to our results presented here. CAT is an oxidative-stress gene. Its mutation weakens the anti-oxidant system in the cochlea, thereby hampering the elimination of the reactive oxygen species generated by noise exposure. 
Consequently, the structure and function of the cochlea are impaired, ultimately causing hearing loss. Furthermore, noise exposure can damage cochlear hair cells and ribbon synapses between hair cells and nerve fibres [29-31]. NRN1 is a small polypeptide closely related to the plasticity of neurites in the human central nervous system. As a neurotrophic factor, NRN1 has multiple effects in the nervous system. It can significantly promote the growth and branch formation of neurites [32] and establishment of functional synapses [33]. Additionally, it is necessary for the survival of neurons [34]. A previous study by our group has shown that a recombinant $N R N 1$ induced extensive neuritogenesis from PC12 cells [35]. Picard et al. have observed that knocking out NRN1 impairs the development and plasticity of excitatory visual cortical networks in mice [36]. Taken together, damages the auditory system more than steady-state noise at the equivalent level $[10,11,37]$. describe the temporal structure of noise, and a mean kurtosis of 10 was used as the boundary value 
considered as the boundary. Noise kurtosis damages the auditory system via direct mechanical force and by disrupting the metabolism [10]. Considering that complex noise is more harmful to the auditory system than steady-state noise, researchers have begun to adjust the energy parameters or exposure time by using kurtosis. For example, Zhao et al. [10] and Goley et al. [38] have proposed correction methods for exposure time and noise energy, respectively. In this study, the correction method for the exposure time was used to adjust the CNE. We observed an association between investigated gene-lifestyle-factor interactions while investigating the effects of noise-metric- 
NIHL. Furthermore, we also found a potential five-locus noise-metric-lifestyle-factor interaction model involving smoking, video volume, physical exercise, working pressure, and kurtosis, as well as a seven-locus model including smoking, video volume, physical exercise, working pressure, kurtosis, CNE, and adj-CNE. These results are similar to the previous results of our group[17]. Previous results showed that there were positive interactions between noise kurtosis with smoking, video volume and physical exercise. However, previous studies analyzed only the interactions between two-category variables via crossover analysis and failed to analyze the effects of $\mathrm{CNE}$ and adj-CNE on the risk of NIHL. Many studies have shown that smoking-induced hearing loss is likely due to vascular changes, including capillary contraction, increased blood viscosity, and cochlear anoxia $[39,40]$. High-volume noise exposure may lead to hearing loss via a mechanism involving reduced cochlear oxygen tension during and after noise exposure [41]. Moreover, lack of exercise affects blood, oxygen, and nutrient flow to the cochlea, leading to the degradation of the stria vascularis (SV). Blood vessels in the SV are essential for transporting necessary factors, such as oxygen and glucose, to the cochlea [42].

This study is superior to previous studies in multiple aspects. First, we firstly focused on the effects of multidimensional interactions on NIHL risk by analyzing 18 variants, three noise metrics, and four lifestyle factors. Second, we identified for the first time that interaction between NRNI rs3805789 and CAT rs7943316 increases NIHL susceptibility. Third, the associations of the interactions among NRN1 rs3805789, CAT rs7943316, and kurtosis with the risk of NIHL was detected for the first time. However, this study had some limitations as well. First, we could not obtain data regarding other important confounding factors, such as hypertension and diabetes, due 
to technical reasons. Second, the analyses of lifestyle factors depended on the recollection of the subjects, which can be unreliable. Third, because the sample size is not large enough, the results obtained from this study should be verified by studies involving larger sample sizes. Finally, this study is an association study, the mechanisms of the gene-gene or gene-environmental-factor interactions should be investigated in future laboratory and clinical studies.

\section{Conclusion}

In conclusion, complex noise, high $\mathrm{CNE}$, high adj-CNE, smoking, high video volume, and sedentary lifestyle are environmental risk factors for NIHL. Concurrence of NRN1 rs3805789 and CAT rs7943316 constitutes a genetic risk factor for NIHL. Complex noise exposure significantly increases the risk of NIHL in subjects with a high genetic risk score. Interactions between genes and lifestyle as well as noise metrics and lifestyle affect the risk of NIHL. These results provide a theoretical basis for screening genetic and environmental risk factors to prevent NIHL.

\section{Abbreviations}

NIHL: Noise-induced hearing loss; CNE: Cumulative noise exposure; adj-CNE: kurtosis-adjusted cumulative noise exposure; EEH: equal-energy hypothesis; ISO: International Standardization Organization; SV: stria vascularis; ROC: receiver operating characteristic; KASP: kompetitive allele specific polymerase chain reaction; Neuritin1: NRN1; Catalase: CAT; Caspase3: CASP3; Caspase 7: CASP7; NADPH Oxidase3: NOX3; Cadherin-23: CDH23; Heat shock protein: HSP; CV: coefficient of variation; OR: Odds ratio; CI: Confidence interval; SD: Standard deviation. 


\section{Acknowledgements}

The authors thank all the participants and institutions for their contribution to this study.

\section{Authors' contributions}

SYL and JRX are joint first authors. SYL oversaw data analysis and wrote the manuscript. JRX edited the article and conducted the statistical analysis. ZL, SL and YQC conducted the study design and revised the manuscript. TYZ, HYW and LWX carried out the experiment. MBZ and LY was responsible for data collection and final manuscript. All authors approved the final manuscript.

\section{Funding}

This work was supported by Zhejiang Key Research and Development Program of China (No.2015C03039; No.20152013A01), Zhejiang Provincial Program for the Cultivation of Highlevel Innovative Health Talents, China; Zhejiang Health Innovative Talent Training Project of China, general scientific research project of Zhejiang Science and Technology Department of China (No. Y201941671).

\section{Availability of data and materials}

Please contact author for data requests.

\section{Ethics approval and consent to participate}

All the participants signed the informed consent form, and the study was approved by the Science Ethics Committee of Hangzhou Normal University (2017LL107). 


\section{Consent for publication}

405

406

Not applicable.

\section{Competing interests}

The authors declare that they have no conflict of interest.

\section{Author details}

1 Medical School, Hangzhou Normal University, Hangzhou 310000, China

2 Ningbo Center for Disease Control and Prevention, Ningbo 315700, China

3 Central people`s hospital of Zhanjiang, Zhanjiang 524000, China

4 Institute of Occupational Health and Radiation Protection, Zhejiang Provincial Center for Disease

Control and Prevention, Hangzhou 310000, China

\section{Author e-mails}

Shuang-Yan LIU: 1sy12135645@163.com, Jia-Rui XIN: 875326032@qq.com, Zheng LI: lizheng@stu.hznu.edu.cn, Song LEI: leisong713@163.com, Ying-Qi CHEN: 807235129@qq.com, Tian-Yu ZHAO: zhaotianyu0225@foxmail.com, Hai-Yan WANG: haiyan1214@126.com, LiangWen XU: 1wxu2006@163.com, Mei-Bian ZHANG: mbzhang@cdc.zj.cn, Lei YANG: yanglei62@hznu.edu.cn. 


\section{References}

1. WHO. Deafness and hearing loss [online] [DB/OL]. Available at: http://www.who.int/media centre/fact sheets/fs300/en/.

2. Chen YL, Hu WJ. Research progress on mechanisms of noise-induced hearingloss. Chinese J Ind Med. 2018;31(6):438-440.

7. Clark WW, Bohne BA, Boettcher FA. Effect of periodic rest on hearing loss and cochlear damage following exposure to noise. J Acoust Soc Am. 1987; 82(4):1253-1264. Erdreich J. A distribution based definition of impulse noise. J Acoust Soc Am. 1986; 79(4):990-998.

9. Pourbakht A, Yamasoba T. Cochlear damage caused by continuous and intermittent noise exposure. Hear Res. 2003; 178(1-2):70-78. Stanbury M, Rafferty AP, Rosenman K. Prevalence of hearing loss and work-related noise-induced hearing

3. Stanbury M, Rafferty AP, Rosenman K. Prevalence of hearing
loss in Michigan. J Occup Environ Med. 2008;50(1):72-79.

4. Shargorodsky J, Curhan SG, Curhan GC, et al. Change in prevalence of hearing loss in US adolescents.

5. Lao XQ, Yu ITS, Au DKK, et al. Noise Exposure and Hearing Impairment among Chinese Restaurant

6. Frederiksen TW, Ramlau-Hansen CH, Stokholm ZA, et al. Noise-Induced Hearing Loss - A Preventable Disease? Results of a 10-Year Longitudinal Study of Workers Exposed to Occupational Noise. Noise Health. 2017; 19(87):103-111. loss in workers exposed to high-level complex noise. Ear Hear. 2010; 31(4):527-532.

11. Davis RI, Qiu W, Heyer NJ, et al. The use of the kurtosis metric in the evaluation of occupational hearing loss in workers in China: Implications for hearing risk assessment. Noise \& Health. 2012; 14(61):330-342. 
Clin Exp Patho. 2015; 8(3):2852-2863.

14. Li YH, Yu SF, Gu GZ, et al. Polymorphisms of heat shock protein 70 genes (HSPA1A, HSPA1B and HSPA1L) and susceptibility of noise-induced hearing loss in a Chinese population: A case-control study. Plos One. 2017;12(2): e0171722..

15. Zhang XH, Ni YQ, Liu Y, et al. Screening of noise-induced hearing loss (NIHL)-associated SNPs and the assessment of its genetic susceptibility. Environ Health-Glob. 2019; 18(1):30.

16. Wu YY, Ni JT, Qi MJ, et al. Associations of genetic variation in CASP3 gene with noise-induced hearing loss in a Chinese population: a case-control study. Environ Health-Glob. 2017; 16(1):78.

17. Zhao TY, Wang YN, Li Z, et al. Associations of noise kurtosis, genetic variations in NOX3 and lifestyle factors with noise-induced hearing loss. Environ Health-Glob. 2020;19(1):1-13.

18. Henderson D, Bielefeld EC, Harris KC, et al. The role of oxidative stress in noise-induced hearing loss. Ear Hearing. 2006; 27(1):1-19.

19. Rybak LP, Whitworth CA, Mukherjea D, et al. Mechanisms of cisplatin-induced ototoxicity and prevention. Hearing Res. 2007; 226(1-2):157-167.

20. Wang XC, Xu PF, Li P, et al. Alterations in gray matter volume due to unilateral hearing loss. Sci Rep-Uk. 2016; 6:25811..

21. Nakajima K, Kanda E, Hosobuchi A, et al. Subclinical hearing loss, longer sleep duration, and cardiometabolic risk factors in Japanese general population. Int J Otolaryngol. 2014;2014:218218.

22. Qiu W, Davis B, Hamernik RP. Hearing loss from interrupted, intermittent, and time varying Gaussian noise exposures: the applicability of the equal energy hypothesis. J Acoust Soc Am. 2007; 121(3):16131620 .

23. Qiu W, Hamernik RP, Davis RI. The value of a kurtosis metric in estimating the hazard to hearing of complex industrial noise exposures. J Acoust Soc Am. 2013; 133(5):2856-2866.

24. Lou XY, Chen GB, Yan L, et al. A generalized combinatorial approach for detecting gene-by-gene and gene-by-environment interactions with application to nicotine dependence. Am J Hum Genet. 2007; 80(6):1125-1137.

25. Chen B, Du Z, Dong X, et al. Association of Variant Interactions in RANK, RANKL, OPG, TRAF6, and NFATC1 Genes with the Development of Osteonecrosis of the Femoral Head. DNA Cell Biol. 2019; 38(7):734-746.

26. Feng C, Yang Y, Yang S, et al. Effect of gene-gene and gene-environment interaction on the risk of firstever stroke and poststroke death. Mol Genet Genomic Med. 2019; 7(8):e846.

27. Zhang LX, Ding RH, Kuang P, et al. Interaction between CONNEXIN37 and PDE4D gene polymorphisms with susceptibility to ischemic stroke in Chinese population. Exp Biol Med. 2019; 244(18):1642-1647.

28. Li J, Wei Z, Hakonarson H. Application of computational methods in genetic study of inflammatory bowel disease. World J Gastroenterol. 2016; 22(3):949-960.

29. Wichmann C, Moser T. Relating structure and function of inner hair cell ribbon synapses. Cell Tissue Res. 2015;361(1):95-114.

30. Moser T, Starr A. Auditory neuropathy - neural and synaptic mechanisms. Nat Rev Neurol. 2016;12(3):135-149.

31. Roux I, Safieddine S, Nouvian R, et al. Otoferlin, defective in a human deafness form, is essential for exocytosis at the auditory ribbon synapse. Cell. 2006; 127(2):277-289.

32. Karamoysoyli E, Burnand RC, Tomlinson DR, et al. Neuritin mediates nerve growth factor-induced axonal regeneration and is deficient in experimental diabetic neuropathy. Diabetes. 2008;57(1):181-189.

33. Fujino T, Leslie JH, Eavri R, et al. CPG15 regulates synapse stability in the developing and adult brain. 
Genes Dev. 2011; 25(24):2674-2685.

34. Sharma TP, Liu Y, Wordinger RJ, et al. Neuritin 1 promotes retinal ganglion cell survival and axonal regeneration following optic nerve crush. Cell Death Dis. 2015; 6:e1661.

35. Zhang P, Luo X, Guo Z, et al. Neuritin Inhibits Notch Signaling through Interacted with Neuralized to

36. Picard N, Leslie JH, Trowbridge SK, et al. Aberrant development and plasticity of excitatory visual cortical

37. Xie HW, Qiu W, Heyer NJ, et al. The Use of the Kurtosis-Adjusted Cumulative Noise Exposure Metric in

39. Hwang JH, Chen JC, Hsu CJ, et al. Plasma reactive oxygen species levels are correlated with severity of

40. Fechter LD, Thorne PR, Nuttall AL. Effects of carbon monoxide on cochlear electrophysiology and blood 
Table 1 Basic Information for 18 SNPs

\begin{tabular}{|c|c|c|c|c|c|}
\hline SNP ID & Gene & Chromosome & Position & Function & Alleles \\
\hline rs1049216 & CASP3 & 4 & 184628935 & 3 Prime UTR Variant & $\mathrm{G} / \mathrm{A}$ \\
\hline rs6948 & CASP3 & 4 & 184627976 & 3 Prime UTR Variant & $\mathrm{T} / \mathrm{G}$ \\
\hline rs3805789 & NRNI & 6 & 6003752 & $\begin{array}{c}\text { Intron Variant, } \\
5 \text { Prime UTR Variant }\end{array}$ & $\mathrm{C} / \mathrm{T}$ \\
\hline rs2227956 & HSPAIL & 6 & 31810495 & Missense Variant & $\mathrm{G} / \mathrm{A}, \mathrm{C}, \mathrm{T}$ \\
\hline rs1043618 & $\begin{array}{l}\text { HSPAIA/ } \\
\text { HSPAIL }\end{array}$ & 6 & 31815730 & $\begin{array}{l}5 \text { Prime UTR Variant, } \\
\text { 2KB Upstream Variant }\end{array}$ & G/A, C, T \\
\hline rs 2763979 & $H S P A I B$ & 6 & 31826815 & 2KB Upstream Variant & $\mathrm{C} / \mathrm{T}$ \\
\hline rs3749930 & NOX3 & 6 & 155440112 & Missense Variant & $\mathrm{G} / \mathrm{T}$ \\
\hline rs12665231 & NOX3 & 6 & 155395463 & 3 Prime UTR Variant & $\mathrm{T} / \mathrm{C}$ \\
\hline rs12195525 & NOX3 & 6 & 155454846 & $\begin{array}{c}\text { Stop Gained, } \\
\text { Synonymous Variant }\end{array}$ & G/A, T \\
\hline rs3752752 & $\mathrm{CDH} 23$ & 10 & 71695444 & Synonymous Variant & $\mathrm{T} / \mathrm{C}$ \\
\hline $\operatorname{rs} 3802711$ & $\mathrm{CDH} 23$ & 10 & 71784329 & Missense Variant & $\mathrm{G} / \mathrm{A}$ \\
\hline rs 1227049 & $\mathrm{CDH} 23$ & 10 & 71675131 & Missense Variant & $\mathrm{G} / \mathrm{A}, \mathrm{C}, \mathrm{T}$ \\
\hline rs12415607 & CASP7 & 10 & 113678445 & 2KB Upstream Variant & $\mathrm{C} / \mathrm{A}$ \\
\hline rs1127687 & CASP7 & 10 & 113730350 & 3 Prime UTR Variant & G/A \\
\hline rs564250 & $C A T$ & 11 & 34437314 & 2KB Upstream Variant & T/A, C \\
\hline rs769214 & $C A T$ & 11 & 34438170 & 2KB Upstream Variant & G/A \\
\hline rs769217 & $C A T$ & 11 & 34461361 & Synonymous Variant & $\mathrm{C} / \mathrm{T}$ \\
\hline rs7943316 & $C A T$ & 11 & 34438925 & 2KB Upstream Variant & $\mathrm{A} / \mathrm{T}$ \\
\hline
\end{tabular}

CASP3: Caspase3; NRN1: Neuritin1; HSP: Heat shock protein; NOX3: NADPH Oxidase3; CDH23: Cadherin-23; CASP7: Caspase7; CAT: Catalase. 
Table 2 General characteristics of subjects between NIHL and control groups

\begin{tabular}{|c|c|c|c|c|c|c|}
\hline \multicolumn{2}{|c|}{ Characteristics } & \multirow[t]{2}{*}{ Total $(n=614)$} & \multirow[t]{2}{*}{ NIHL (n=307) } & \multirow[t]{2}{*}{ Control $(\mathbf{n}=307)$} & \multirow[t]{2}{*}{$\chi^{2} / \mathrm{z}$} & \multirow[t]{2}{*}{$\boldsymbol{P}$} \\
\hline Sex, n $(\%)$ & & & & & & \\
\hline & Male & $474(77.2)$ & $237(38.6)$ & 237 (38.6) & 000 & 1000 \\
\hline & Female & $140(22.8)$ & $70(11.4)$ & $70(11.4)$ & 0.000 & 1.000 \\
\hline Age, y & & $35(30-43)$ & $36(30-43)$ & $34(30-42)$ & -1.959 & 0.050 \\
\hline \multicolumn{7}{|l|}{ Education, n $(\%)$} \\
\hline & High school and above & $347(56.5)$ & $168(27.4)$ & $179(29.2)$ & & \\
\hline & $\begin{array}{l}\text { Junior high school and } \\
\text { below }\end{array}$ & $267(43.5)$ & $139(22.6)$ & $128(20.8)$ & 0.802 & 0.371 \\
\hline Years of noise exposure, $y$ & & $3.00(1.43-6.00)$ & $3.00(1.20-6.00)$ & $3.00(2.00-6.00)$ & -1.002 & 0.317 \\
\hline $\mathrm{HTL}_{346}, \mathrm{~dB}$ & & $25.00(17.83-36.96)$ & $36.83(29.83-49.83)$ & $17.83(14.17-21.00)$ & -21.442 & $<0.001$ \\
\hline \multirow[t]{3}{*}{ Kurtosis, median $\left(\mathrm{P}_{25} \sim \mathrm{P}_{75}\right)$} & & $6.62(4.28-13.15)$ & $7.25(4.63-14.30)$ & $5.85(4.06-12.51)$ & -2.755 & 0.006 \\
\hline & $<4$ & $114(18.6)$ & $47(7.7)$ & $67(10.9)$ & \multirow{2}{*}{4.039} & \multirow{2}{*}{$\mathbf{0 . 0 3 8}$} \\
\hline & $\geq 4$ & $500(81.4)$ & $260(42.3)$ & $240(39.1)$ & & \\
\hline \multirow[t]{3}{*}{$\begin{array}{c}\text { CNE, median }\left(\mathrm{P}_{25} \sim \mathrm{P}_{75}\right), \\
\mathrm{dB}(\mathrm{A})\end{array}$} & & 93.69 (89.48-97.57) & $93.73(89.67-98.24)$ & $93.62(89.07-96.88)$ & -1.23 & 0.219 \\
\hline & $<97.1420$ & $449(73.1)$ & $213(34.7)$ & $236(38.4)$ & 4.384 & 0.036 \\
\hline & $\geq 97.1420$ & $165(26.9)$ & $94(15.3)$ & 71 (11.6) & & \\
\hline \multirow[t]{3}{*}{$\begin{array}{c}\text { Adj-CNE, median }\left(\mathrm{P}_{25} \sim \mathrm{P}_{75}\right), \\
\mathrm{dB}(\mathrm{A})\end{array}$} & & $94.89(90.02-99.23)$ & $95.34(90.20-99.61)$ & $94.61(89.75-98.59)$ & -1.564 & 0.118 \\
\hline & $<96.9939$ & $387(63.0)$ & $178(29.0)$ & $209(34.0)$ & \multirow{2}{*}{6.717} & \multirow{2}{*}{0.010} \\
\hline & $\geq 96.9939$ & $227(37.0)$ & $129(21.0)$ & $98(16.0)$ & & \\
\hline \multicolumn{7}{|l|}{ Smoking, n (\%) } \\
\hline & No & $325(52.9)$ & $150(24.4)$ & $175(28.5)$ & \multirow{2}{*}{4.086} & \multirow{2}{*}{0.043} \\
\hline & Yes & $289(47.1)$ & $157(25.6)$ & $132(21.5)$ & & \\
\hline \multicolumn{7}{|l|}{ Video volume, n (\%) } \\
\hline & Low & $149(24.3)$ & $60(9.8)$ & $89(14.5)$ & 7.453 & 0.006 \\
\hline
\end{tabular}


$\begin{array}{lll}\text { High } & 465(75.7) & 247(40.2)\end{array}$

Physical exercise, n (\%)

\begin{tabular}{cccccc} 
Never & $418(68.1)$ & $224(36.5)$ & $194(31.6)$ & 6.745 & $\mathbf{0 . 0 0 9}$ \\
Regular & $196(31.9)$ & $83(13.5)$ & $113(18.4)$ & & \\
& & & & & \\
Low & $113(18.4)$ & $49(8.0)$ & $64(10.4)$ & 2.440 & 0.118 \\
High & $501(81.6)$ & $258(42.0)$ & $243(39.6)$ & & \\
\hline
\end{tabular}

556

557

558 
Table 3 The genotype and allele frequencies of 18 SNPs in NIHL cases and control subjects

\begin{tabular}{|c|c|c|c|c|c|c|c|c|c|c|c|c|}
\hline SNP ID & Gene & Group & \multicolumn{3}{|c|}{ Genotype (frequency) } & \multicolumn{2}{|c|}{ Allele (frequency) } & \multirow[t]{2}{*}{$P$} & \multirow[t]{2}{*}{ OR $(95 \% \mathrm{CI})$} & \multirow[t]{2}{*}{$\mathrm{MAF}^{\mathrm{a}}$} & \multirow[t]{2}{*}{$\mathrm{MAF}^{\mathrm{b}}$} & \multirow[t]{2}{*}{ HWE $\mathrm{p}$} \\
\hline \multirow{3}{*}{ rs1049216 } & \multirow{3}{*}{ CASP3 } & & AA & $\mathrm{AG}$ & GG & A & G & & & & & \\
\hline & & Case & $10(3.3)$ & $105(34.2)$ & $192(62.5)$ & $125(20.4)$ & $489(79.6)$ & \multirow{2}{*}{0.046} & 1.046 & \multirow{2}{*}{0.403} & \multirow{2}{*}{0.160} & \multirow{2}{*}{0.616} \\
\hline & & Control & $9(2.9)$ & $80(26.1)$ & $218(71.0)$ & $98(16.0)$ & $516(84.0)$ & & $(1.005-1.802)$ & & & \\
\hline \multirow{3}{*}{ rs6948 } & \multirow{3}{*}{ CASP3 } & & GG & GT & $\mathrm{TT}$ & G & $\mathrm{T}$ & & & & & \\
\hline & & Case & $10(3.3)$ & $92(30.0)$ & $205(66.8)$ & $112(18.2)$ & $502(81.8)$ & \multirow{2}{*}{0.064} & $1.334(0.982-$ & \multirow{2}{*}{0.426} & \multirow{2}{*}{0.143} & \multirow{2}{*}{0.431} \\
\hline & & Control & $8(2.6)$ & $72(23.5)$ & 227 (73.9) & $88(14.3)$ & $526(85.7)$ & & $1.809)$ & & & \\
\hline \multirow{3}{*}{ rs3805789 } & \multirow{3}{*}{ NRN1 } & & $\mathrm{CC}$ & $\mathrm{CT}$ & $\mathrm{TT}$ & $\mathrm{C}$ & $\mathrm{T}$ & \multirow{3}{*}{0.864} & & & & \\
\hline & & Case & $84(27.4)$ & $163(53.1)$ & $60(19.5)$ & $331(53.9)$ & $283(46.1)$ & & 1.020 & \multirow{2}{*}{0.300} & \multirow[t]{2}{*}{0.466} & \multirow[t]{2}{*}{0.216} \\
\hline & & Control & $93(30.3)$ & $142(46.3)$ & $72(23.5)$ & $328(53.4)$ & $286(46.6)$ & & $(0.815-1.276)$ & & & \\
\hline \multirow{3}{*}{ rs2227956 } & \multirow{3}{*}{ HSPAIL } & & $\mathrm{CC}$ & $\mathrm{CT}$ & TT & $\mathrm{C}$ & $\mathrm{T}$ & & & & & \\
\hline & & Case & $15(4.9)$ & $86(28.0)$ & $206(67.1)$ & $116(18.9)$ & $498(81.1)$ & \multirow{2}{*}{0.942} & 0.989 & \multirow{2}{*}{0.123} & \multirow{2}{*}{0.191} & \multirow{2}{*}{0.671} \\
\hline & & Control & $10(3.3)$ & 97 (31.6) & $200(65.1)$ & $117(19.1)$ & 497 (80.9) & & $(0.744-1.316)$ & & & \\
\hline & & & $\mathrm{CC}$ & CG & GG & $\mathrm{C}$ & G & & & & & \\
\hline rs1043618 & НDPAIA & Case & $42(13.7)$ & $118(38.4)$ & 147 (47.9) & $202(32.9)$ & $412(67.1)$ & & 0.985 & 0.481 & 0.332 & 0.587 \\
\hline & & Control & $36(11.7)$ & $132(43.0)$ & $139(45.3)$ & $204(33.2)$ & $410(66.8)$ & & $(0.777-1.250)$ & & & \\
\hline & & & $\mathrm{CC}$ & $\mathrm{CT}$ & $\mathrm{TT}$ & $\mathrm{C}$ & $\mathrm{T}$ & & & & & \\
\hline rs2763979 & HSPAIB & Case & $168(54.7)$ & $102(33.2)$ & $37(12.1)$ & 438 (71.3) & $176(28.7)$ & 0153 & 1.098 & (448 & 0.306 & 0.387 \\
\hline & & Control & $151(49.2)$ & $124(40.4)$ & $32(10.4)$ & $426(69.4)$ & $188(30.6)$ & $0.4 J 3$ & $(0.860-1.403)$ & $0.4+0$ & & \\
\hline & & & GG & GT & $\mathrm{TT}$ & G & $\mathrm{T}$ & & & & & \\
\hline rs3749930 & $N O X 3$ & Case & $86(28.0)$ & $148(48.2)$ & $73(23.8)$ & $320(52.1)$ & 294 (47.9) & (17) & 1.192 & 07 & 177 & 0660 \\
\hline & & Control & $68(22.1)$ & $157(51.1)$ & $82(26.7)$ & $293(47.7)$ & $321(52.3)$ & $0.12 J$ & $(0.953-1.492)$ & 0.191 & 0.411 & 0.002 \\
\hline rs 12665231 & NOX3 & & $\mathrm{CC}$ & CT & TT & $\mathrm{C}$ & $\mathrm{T}$ & & & & & \\
\hline
\end{tabular}




\begin{tabular}{|c|c|c|c|c|c|c|c|c|c|c|c|c|}
\hline & & Case & $23(7.5)$ & $100(32.6)$ & $184(59.9)$ & $146(23.8)$ & $468(76.2)$ & 0.841 & 0.974 & 0.188 & 0.243 & 0.126 \\
\hline & & Control & $23(7.5)$ & $103(33.6)$ & $181(59.0)$ & $149(24.3)$ & $465(75.7)$ & & $(0.749-1.265)$ & & & \\
\hline \multirow{3}{*}{ rs12195525 } & \multirow{3}{*}{$N O X 3$} & & GG & TG & $\mathrm{TT}$ & $\mathrm{G}$ & $\mathrm{T}$ & & & \multirow{3}{*}{0.129} & \multirow{3}{*}{0.104} & \multirow{3}{*}{0.103} \\
\hline & & Case & $267(87.0)$ & $35(11.4)$ & $5(1.6)$ & $569(92.7)$ & $45(7.3)$ & \multirow{2}{*}{0.057} & 1.471 & & & \\
\hline & & Control & $249(81.1)$ & $52(16.9)$ & $6(2.0)$ & $550(89.6)$ & $64(10.4)$ & & $(0.987-2.193)$ & & & \\
\hline \multirow{3}{*}{ rs 3752752} & \multirow{3}{*}{$\mathrm{CDH} 23$} & & $\mathrm{CC}$ & $\mathrm{TC}$ & $\mathrm{TT}$ & $\mathrm{C}$ & $\mathrm{T}$ & & & & & \\
\hline & & Case & $84(27.4)$ & $142(46.3)$ & $81(26.4)$ & $310(50.5)$ & $304(49.5)$ & \multirow{2}{*}{0.954} & 0.994 & \multirow{2}{*}{0.445} & \multirow{2}{*}{0.493} & \multirow{2}{*}{0.154} \\
\hline & & Control & 85 (27.7) & $141(45.9)$ & $81(26.4)$ & $311(50.7)$ & $303(49.3)$ & & $(0.794-1.243)$ & & & \\
\hline \multirow{3}{*}{ rs 3802711} & \multirow{3}{*}{$\mathrm{CDH} 23$} & & AA & $\mathrm{CA}$ & $\mathrm{CC}$ & A & $\mathrm{C}$ & & & & & \\
\hline & & Case & $19(6.2)$ & $107(34.9)$ & $181(59.0)$ & 145 (23.6) & $469(76.4)$ & \multirow{2}{*}{0.305} & 1.151 & \multirow{2}{*}{0.139} & \multirow{2}{*}{0.212} & \multirow{2}{*}{0.34} \\
\hline & & Control & $11(3.6)$ & $108(35.2)$ & $188(61.2)$ & $130(21.2)$ & $484(78.8)$ & & $(0.880-1.506)$ & & & \\
\hline \multirow{3}{*}{ rs1227049 } & \multirow{3}{*}{$\mathrm{CDH} 23$} & & $\mathrm{CC}$ & CG & GG & $\mathrm{C}$ & G & & & & & \\
\hline & & Case & $37(12.1)$ & $127(41.4)$ & $143(46.6)$ & $201(32.7)$ & $413(67.3)$ & \multirow{2}{*}{0.325} & 1.129 & \multirow{2}{*}{0.185} & \multirow{2}{*}{0.301} & \multirow{2}{*}{0.612} \\
\hline & & Control & $26(8.5)$ & $133(43.3)$ & $148(48.2)$ & $185(30.1)$ & $429(69.9)$ & & $(0.887-1.436)$ & & & \\
\hline \multirow{3}{*}{ rs12415607 } & \multirow{3}{*}{ CASP7 } & & AA & $\mathrm{CA}$ & $\mathrm{CC}$ & A & $\mathrm{C}$ & & & & & \\
\hline & & Case & $51(16.6)$ & $155(50.5)$ & $101(32.9)$ & $257(41.90$ & 357 (58.1) & \multirow{2}{*}{0.035} & 1.280 & \multirow{2}{*}{0.268} & \multirow{2}{*}{0.360} & \multirow{2}{*}{0.237} \\
\hline & & Control & $35(11.4)$ & $151(49.2)$ & $121(39.4)$ & $221(36.0)$ & $393(64.0)$ & & $(1.017-1.611)$ & & & \\
\hline & & & AA & GA & GG & A & G & & & & & \\
\hline rs1127687 & CASP7 & Case & $17(5.5)$ & $94(30.6)$ & $196(63.8)$ & $128(20.8)$ & 486 (79.2) & 0103 & 0.801 & $0>230$ & (2) & 0955 \\
\hline & & Control & $19(5.5)$ & 114 (37.1) & $174(56.7)$ & $152(24.8)$ & $462(75.2)$ & 0.100 & $(0.613-1.046)$ & 0.250 & $0.2+10$ & 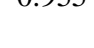 \\
\hline & & & $\mathrm{CC}$ & $\mathrm{TC}$ & TT & $\mathrm{C}$ & $\mathrm{T}$ & & & & & \\
\hline rs 564250 & CAT & Case & $195(63.5)$ & $92(30.0)$ & $20(6.5)$ & 482 (78.5) & $132(21.5)$ & $0^{\circ}$ & 0.933 & 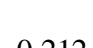 & ( 00 & 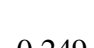 \\
\hline & & Control & $198(64.5)$ & $93(30.3)$ & $16(5.2)$ & 489 (79.6) & $125(20.4)$ & 0.623 & $(0.709-1.229)$ & 0.212 & 0.204 & 0.249 \\
\hline & & & AA & AG & GG & A & G & & & & & \\
\hline rs769214 & CAT & Case & $31(10.1)$ & $120(39.1)$ & $156(50.8)$ & $182(29.6)$ & $432(70.4)$ & 0852 & 0.977 & 0,473 & 0301 & 0972 \\
\hline & & Control & $28(9.1)$ & $129(42.0)$ & $150(48.9)$ & $185(30.1)$ & $429(69.9)$ & & $(0.765-1.247)$ & & & \\
\hline
\end{tabular}




\begin{tabular}{|c|c|c|c|c|c|c|c|c|c|c|c|c|}
\hline \multirow{3}{*}{ rs769217 } & \multirow{3}{*}{$C A T$} & & $\mathrm{CC}$ & CT & TT & $\mathrm{C}$ & $\mathrm{T}$ & \multirow{3}{*}{0.864} & \multirow{3}{*}{$\begin{array}{c}1.020 \\
(0.815-1.275)\end{array}$} & \multirow{3}{*}{0.263} & \multirow{3}{*}{0.493} & \multirow{3}{*}{0.333} \\
\hline & & Case & $83(27.0)$ & $148(48.2)$ & $76(24.8)$ & $314(51.1)$ & $300(48.9)$ & & & & & \\
\hline & & Control & $83(27.0)$ & $145(47.2)$ & $79(25.7)$ & $311(50.7)$ & $303(49.3)$ & & & & & \\
\hline \multirow{3}{*}{ rs7943316 } & \multirow{3}{*}{$C A T$} & & AA & AT & TT & A & $\mathrm{T}$ & & & & & \\
\hline & & Case & $160(52.1)$ & $112(36.5)$ & $35(11.4)$ & $432(70.4)$ & $182(29.6)$ & \multirow{2}{*}{0.664} & 1.056 & \multirow{2}{*}{0.488} & \multirow{2}{*}{0.308} & \multirow{2}{*}{0.609} \\
\hline & & Control & 149 (48.5) & 127 (41.4) & $31(10.1)$ & $425(69.2)$ & $189(30.8)$ & & $(0.827-1.347)$ & & & \\
\hline
\end{tabular}

MAF: Minor allele frequency; HWE: Hardy-Weinberg equilibrium; a: 1000 genomes; b: Data form this study; OR: odds ratio; CI: confidence interval.

$P$-values of deviation from HWE between the NIHL group and control group. 
Table 4 Association of Multidimensional Gene-Gene Interactions of 18 SNPs with NIHL Risk

\begin{tabular}{|c|c|c|c|c|c|}
\hline No. of loci & Model & TRBA & TEBA & $\mathrm{P}$ value & CVC \\
\hline 1 & $\mathrm{X} 1$ & 0.5480 & 0.5112 & $7(0.1719)$ & $8 / 10$ \\
\hline 2 & X3 X18 & 0.5921 & 0.5771 & $9(0.0107)$ & $10 / 10$ \\
\hline 3 & X3 X10 X16 & 0.6186 & 0.5116 & $6(0.3770)$ & $3 / 10$ \\
\hline 4 & X3 X8 X10 X18 & 0.6662 & 0.4591 & $2(0.9893)$ & $2 / 10$ \\
\hline 5 & X3 X6 X10 X13 X17 & 0.7392 & 0.4650 & $4(0.8281)$ & $3 / 10$ \\
\hline 6 & X3 X5 X10 X12 X13 X17 & 0.8290 & 0.5218 & $7(0.1719)$ & $8 / 10$ \\
\hline 7 & X3 X5 X7 X10 X11 X12 X17 & 0.9060 & 0.5538 & $6(0.3770)$ & $9 / 10$ \\
\hline 8 & X3 X5 X7 X10 X11 X12 X13 X17 & 0.9527 & 0.4351 & $2(0.9893)$ & $4 / 10$ \\
\hline 9 & X3 X4 X5 X7 X8 X10 X12 X13 X17 & 0.9927 & $\mathrm{NaN}$ & $4(0.8281)$ & $4 / 10$ \\
\hline 10 & X3 X4 X5 X7 X8 X10 X11 X12 X13 X17 & 0.9926 & $\mathrm{NaN}$ & $4(0.8281)$ & $3 / 10$ \\
\hline 11 & X2 X3 X4 X5 X6 X7 X8 X11 X12 X14 X17 & 0.9981 & $\mathrm{NaN}$ & $3(0.9453)$ & $1 / 10$ \\
\hline 12 & X1 X3 X4 X5 X6 X7 X8 X10 X12 X13 X14 X17 & 1.0000 & $\mathrm{NaN}$ & $2(0.9893)$ & $1 / 10$ \\
\hline 13 & X1 X2 X3 X4 X5 X7 X8 X9 X11 X12 X14 X16 X17 & 1.0000 & $\mathrm{NaN}$ & $3(0.9453)$ & $2 / 10$ \\
\hline 14 & X1 X2 X3 X4 X5 X6 X7 X8 X9 X11 X12 X14 X16 X17 & 1.0000 & $\mathrm{NaN}$ & $4(0.8281)$ & $4 / 10$ \\
\hline 15 & X1 X2 X3 X4 X5 X6 X7 X8 X9 X10 X11 X12 X13 X14 X18 & 1.0000 & $\mathrm{NaN}$ & $2(0.9893)$ & $8 / 10$ \\
\hline 16 & X1 X2 X3 X4 X5 X6 X7 X8 X9 X10 X11 X12 X13 X14 X15 X18 & 1.0000 & $\mathrm{NaN}$ & $0(1.0000)$ & $8 / 10$ \\
\hline 17 & X1 X2 X3 X4 X5 X6 X7 X8 X9 X10 X11 X12 X13 X14 X15 X16 X17 & 1.0000 & N/A & $0(1.0000)$ & $10 / 10$ \\
\hline 18 & X1 X2 X3 X4 X5 X6 X7 X8 X9 X10 X11 X12 X13 X14 X15 X16 X17 X18 & 1.0000 & N/A & $0(1.0000)$ & $10 / 10$ \\
\hline
\end{tabular}

$P$-values were obtained from the GMDR analysis which adjusted for age, gender, education, years of exposure, kurtosis, CNE, adj-CNE, smoking, video volume, physical exercise, and working pressure. TRBA: Training balanced accuracy; TEBA: Testing Balanced accuracy; CVC: cross-validation consistency; X1: CASP3 rs 1049216; X2: CASP3 rs6948; X3: NRN1 rs3805789; X4: HSPA1L rs2227956; X5: HSPA1A/HSPA1L rs1043618; X6: HSPA1B rs2763979; X7: NOX3 rs3749930; X8: NOX3 rs12665231; X9: NOX3 rs12195525; X10: CDH23 rs3752752; X11: CDH23 rs3802711; X12: CDH23 rs1227049; X13: CASP7 rs12415607; X14: CASP7 rs1127687; X15: CAT rs564250; X16: CAT rs769214; X17: CAT rs769217; X18: CAT rs7943316. 
Table 5 Associations of interactions among genes, noise metrics and lifestyle factors with the risk of NIHL

\begin{tabular}{|c|c|c|c|c|c|}
\hline No. of loci & Model & TRBA & TEBA & $\mathrm{P}$ value & $\mathrm{CVC}$ \\
\hline \multicolumn{6}{|c|}{ A. Gene-noise-metric interaction ${ }^{\mathrm{a}}$} \\
\hline 1 & K4 & 0.5363 & 0.4907 & $5(0.6230)$ & $4 / 10$ \\
\hline 2 & X3 X18 & 0.5903 & 0.5762 & $9(0.0107)$ & $10 / 10$ \\
\hline 3 & X3 X18 K4 & 0.6003 & 0.5863 & $10(0.0010)$ & $10 / 10$ \\
\hline 4 & X3 X18 adj-CNE K4 & 0.6125 & 0.5856 & $9(0.0107)$ & $10 / 10$ \\
\hline 5 & X3 X18 adj-CNE CNE K4 & 0.6125 & 0.5856 & $9(0.0107)$ & $10 / 10$ \\
\hline \multicolumn{6}{|c|}{ B. Gene-lifestyle-factor interaction ${ }^{\mathrm{b}}$} \\
\hline 1 & $\mathrm{Y} 3$ & 0.5554 & 0.5356 & $6(0.3770)$ & $9 / 10$ \\
\hline 2 & X3 X18 & 0.5927 & 0.5349 & $7(0.1719)$ & $7 / 10$ \\
\hline 3 & $\mathrm{X} 3 \mathrm{X} 18 \mathrm{Y} 4$ & 0.6291 & 0.5377 & $9(0.0107)$ & $5 / 10$ \\
\hline 4 & X3 X18 Y1 Y3 & 0.6830 & 0.5850 & $9(0.0107)$ & $9 / 10$ \\
\hline 5 & X3 X18 Y1 Y3 Y4 & 0.7387 & 0.5570 & $9(0.0107)$ & $7 / 10$ \\
\hline 6 & X3 X18 Y1 Y2 Y3 Y4 & 0.7946 & 0.5866 & $9(0.0010)$ & $10 / 10$ \\
\hline \multicolumn{6}{|c|}{ C. Noise-metric-lifestyle-factor interaction ${ }^{c}$} \\
\hline 1 & Y3 & 0.5543 & 0.5340 & $6(0.3770)$ & $9 / 10$ \\
\hline 2 & Y2 Y3 & 0.5816 & 0.5209 & $7(0.1719)$ & $5 / 10$ \\
\hline 3 & Y2 Y3 Y4 & 0.6069 & 0.4792 & $3(0.9453)$ & $6 / 10$ \\
\hline 4 & Y1 Y2 Y3 Y4 & 0.6494 & 0.5509 & $9(0.0107)$ & $10 / 10$ \\
\hline 5 & Y1 Y2 Y3 Y4 K4 & 0.6778 & 0.5467 & $9(0.0107)$ & $10 / 10$ \\
\hline 6 & Y1 Y2 Y3 Y4 K4 adj-CNE & 0.6970 & 0.5437 & $9(0.0107)$ & $7 / 10$ \\
\hline 7 & Y1 Y2 Y3 Y4 K4 adj-CNE CNE & 0.6920 & 0.5503 & $9(0.0107)$ & $10 / 10$ \\
\hline
\end{tabular}

${ }^{a}$ Adjusted for age, gender, education, smoking, video volume, physical exercise, and working pressure;

${ }^{\mathrm{b}}$ Adjusted for age, gender, education, years of exposure, kurtosis, CNE, adj-CNE;

${ }^{c}$ Adjusted for age, gender, education;

TRBA: Training balanced accuracy; TEBA: Testing Balanced accuracy; CVC: cross-validation consistency; X3: NRN1 rs3805789; X18: CAT rs7943316; K4: kurtosis; Y1: smoking; Y2: video volume; Y3: physical exercise; Y4: working pressure. 


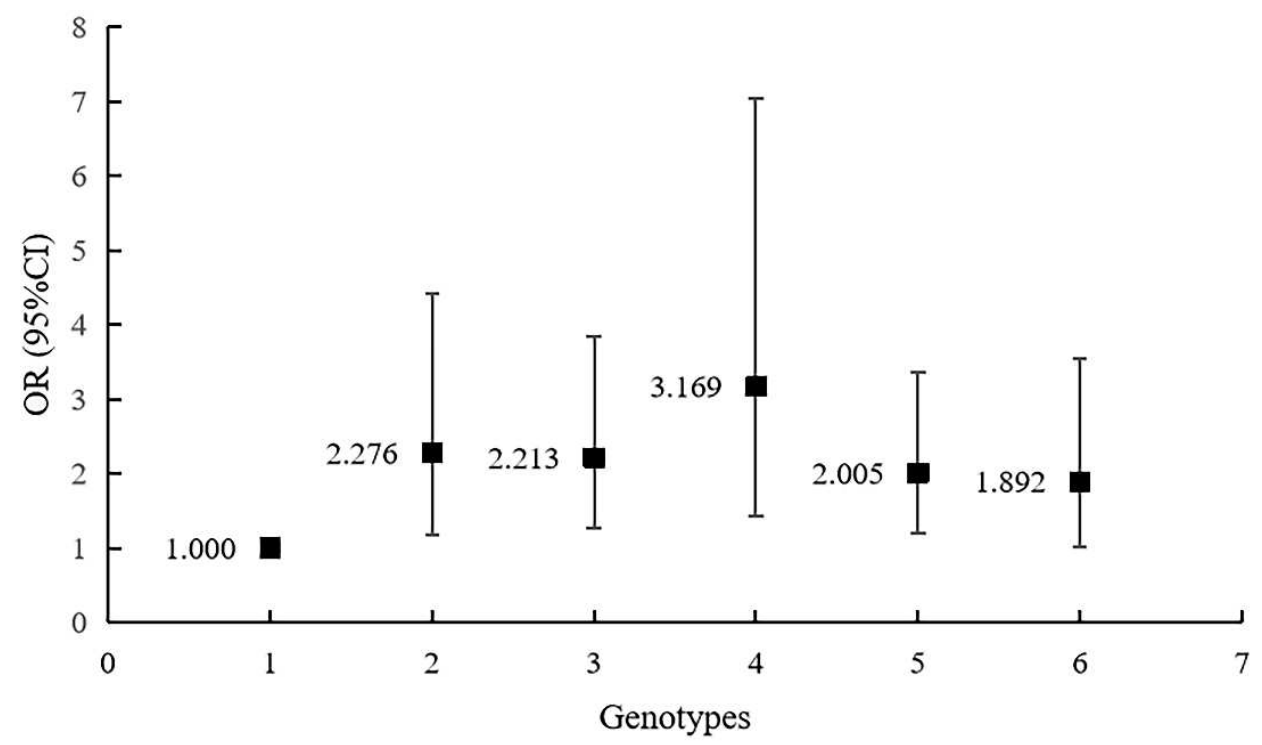

Figure 1 Stratified analysis for gene-gene interaction on NIHL risk using logistic regression.

The odds ratios (ORs) were calculated after adjustment for age, gender, education, years of exposure, kurtosis, CNE, adj-CNE, smoking, video volume, physical exercise, and working pressure. 1: rs3805789-CC and rs7943316-AA; 2: rs3805789-CC and rs7943316-AT; 3: rs3805789-CT and rs7943316-AA; 4: rs3805789-CT and rs7943316-TT; 5: rs3805789-CT/TT and rs7943316-AA; 6: rs3805789-CC and rs7943316-AA/TT.

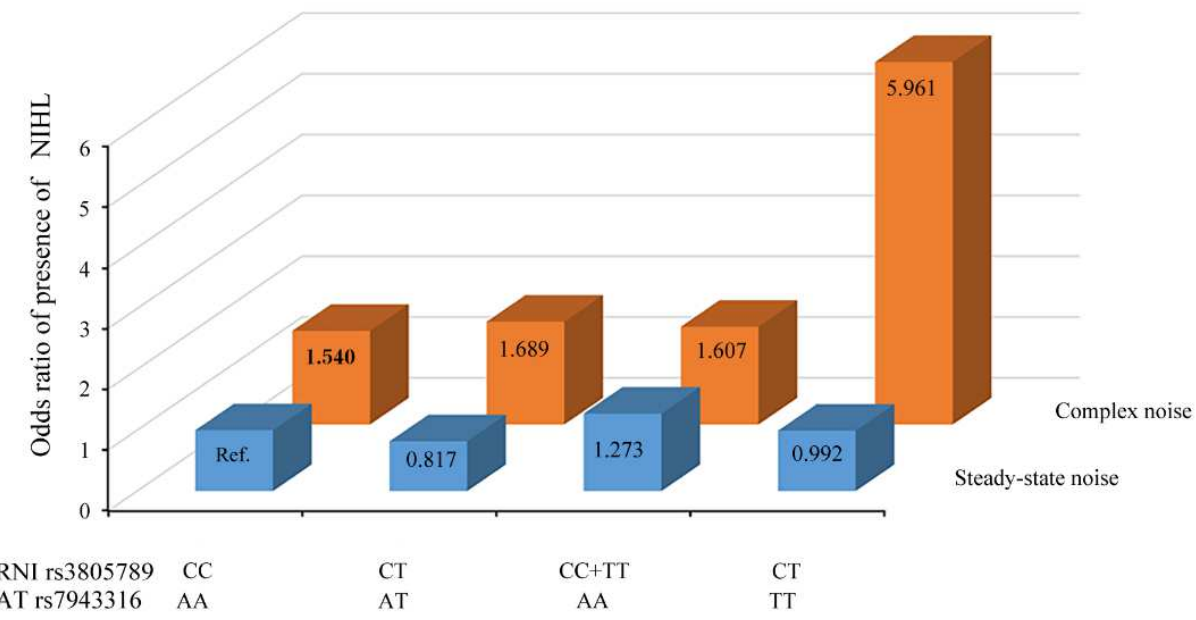

Figure 2 Joint effects of the NRN1 rs3805789 and CAT rs7943316 with kurtosis on NIHL risk. The reference group was defined as subjects exposed steady-state noise who carry NRN1 rs3805789 CC and CAT rs7943316 AA. Ref: reference group. The odds ratios (ORs) were calculated by the logistic regression analysis after adjustment for age, gender, education, years of exposure, smoking, video volume, physical exercise, and working pressure. 
Table S1 Primer information A

\begin{tabular}{cccc}
\hline SNP ID & Primer Allele FAM & Primer Allele HEX & Primer Common \\
\hline rs1049216 & AGTAATTGTGAAAAAGTTAAACATTGAAGTAAT & AGTAATTGTGAAAAAGTTAAACATTGAAC & CAGTCTTAAGTGGGGGGAATATCATAAAA \\
rs6948 & GGAGGCCAGAGCTGAGCC & CGGAGGCCAGAGCTGAGCA & AGCCTGCCTCCCGGGCTGA \\
rs3805789 & TTCCGCCCTGAGGCCGAC & CTTTCCGCCCTGAGGCCGAT & CACTCAGCTTCCAGCCGGGTT \\
rs2227956 & ATGGTATTCTCAATGTCACAGCCAC & AATGGTATTCTCAATGTCACAGCCAT & CTTGTTCACCTTGCCGGTGCTCTT \\
rs1043618 & CCTGCTCTCTGTCGGCTCC & CCTGCTCTCTGTCGGCTCG & GTGTTCCGTTTCCAGCCCCCAA \\
rs2763979 & ATTCCTGGTTCACTCCTGGACG & AATTCCTGGTTCACTCCTGGACA & CTCGGGACTGTGAGGTCCTACTT \\
rs3749930 & ACACAACCACTGAATTGCTAAGGAC & AACACAACCACTGAATTGCTAAGGAA & CAGACCGGTGACGCCTGCTATT \\
rs12665231 & AGCTAAGGAAGTATGGTATAGTGTACT & GCTAAGGAAGTATGGTATAGTGTACC & CCCAGTTGGGAACTGGTGAATAATAATTA \\
rs12195525 & GTTCCTCTTATGAATGAAATAAGGTTCA & GTTCCTCTTATGAATGAAATAAGGTTTCG & CTGTGCCTGAATTTTAACTGCATGCTAAT \\
rs3752752 & AAACATCACCCTCCTGGACATCAAT & CATCACCCTCCTGGACATCAAC & GTCCTTCCACGTGGGGTGGTT \\
rs3802711 & GTCCAGCTCCACGTCCTTCC & GGTCCAGCTCCACGTCCTTCT & TCTCCTGTGGAGGGGGTGCTAA \\
rs1227049 & AGCTGACTTCCCCAAAGGTGG & AGCTGACTTCCCCAAAGGTGC & GCTGTTGCAGGCAACTGACAATGAT \\
rs12415607 & AATGGAGTACATGCTTAGTGGTCG & GAATGGAGTACATGCTTAGTGGTCT & AGAAGATGGCGTTCTTGCCTGGTAT \\
rs1127687 & CACTCCATCTCAGTCAGTGGC & CTCACTCCATCTCAGTCAGTGGT & TGGAAAATGGAGCCATGACAAGAACAAA \\
rs564250 & AGACCTGGAGTCATTTCTTAACCTTTA & GACCTGGAGTCATTTCTTAACCTTTG & CAAATCCATAGTAACAAAAACAAAGGAAAA \\
rs769214 & TTTCAAAATTCCTGCTTACCTGGGA & TCAAAATTCCTGCTTACCTGGGG & ACTGGAGAAATCTGCTTCCCCAAATTTTA \\
rs769217 & GTGGCCAACTACCAGCGTGAC & AGTGGCCAACTACCAGCGTGAT & TACCCTGATTGTCCTGCATGCACAT \\
rs7943316 & AAATCTGCCTGTTGCCCCGAGT & AAATCTGCCTGTTGCCCCGAGA & GGTGCTGATTGGCTGAGCCTGAA \\
\hline
\end{tabular}


Table S2 Primer information B

\begin{tabular}{|c|c|c|c|c|}
\hline SNP ID & Sequence & CG\%_FAM & CG\%_HEX & CG\% Common \\
\hline rs1049216 & GGGGAATATCATAAAAATTC[A/G] TTACTTCAATGTTTAACTTT & 21.2 & 24.2 & 37.9 \\
\hline rs6948 & AGCCTGCCTCCCGGGCTGAG[G/T] GCTCAGCTCTGGCCTCCGGC & 72.2 & 68.4 & 73.7 \\
\hline rs3805789 & TCTTTCCGCCCTGAGGCCGA[C/T] GAACCCGGCTGGAAGCTGAG & 70 & 61.9 & 61.9 \\
\hline rs2227956 & TATTCTCAATGTCACAGCCA[C/T] GGACAAGAGCACCGGCAAGG & 44 & 38.5 & 54.2 \\
\hline rs 1043618 & CCAGCCCCCAATCTCAGAGC[C/G] GAGCCGACAGAGAGCAGGGA & 68.4 & 68.4 & 59.1 \\
\hline rs2763979 & GAGGTCCTACTTCTACACAC[C/T] GTCCAGGAGTGAACCAGGAA & 54.5 & 47.8 & 56.5 \\
\hline rs3749930 & GACCGGTGACGCCTGCTATT[G/T] TCCTTAGCAATTCAGTGGTT & 44 & 38.5 & 59.1 \\
\hline rs12665231 & GGAAGTATGGTATAGTGTAC[T/C] GTTCACAATAGTTAATTATT & 37 & 42.3 & 37.9 \\
\hline rs12195525 & TATGAATGAAATAAGGTTTC[A/G] ACTGACAGGTATTAGAATTA & 30 & 32.3 & 37.9 \\
\hline rs 3752752 & ATCACCCTCCTGGACATCAA[T/C] GACAACCACCCCACGTGGAA & 42.3 & 54.5 & 61.9 \\
\hline rs3802711 & GGAGGGGGTGCTAAGGGTCC[G/A] GAAGGACGTGGAGCTGGACC & 65 & 61.9 & 59.1 \\
\hline rs1227049 & GGCAACTGACAATGATGCAG[C/G] CACCTTTGGGGAAGTCAGCT & 54.5 & 54.5 & 48 \\
\hline rs12415607 & TGGCGTTCTTGCCTGGTATC[C/A] GACCACTAAGCATGTACTCC & 45.8 & 42.3 & 48 \\
\hline rs1127687 & GGAGCCATGACAAGAACAAA[G/A] CCACTGACTGAGATGGAGTG & 58.3 & 52.2 & 39.3 \\
\hline rs564250 & GCTTTTTTTTATATAATGAA[C/T] AAAGGTTAAGAAATGACTCC & 37 & 42.3 & 26.7 \\
\hline rs769214 & AAAATTCCTGCTTACCTGGG[A/G] GTAAAATTTGGGGAAGCAGA & 49.2 & 44 & 37.9 \\
\hline rs769217 & GTGGCCAACTACCAGCGTGA[C/T] GGCCCGATGTGCATGCAGGA & 61.9 & 54.5 & 48 \\
\hline rs7943316 & GAGCCTGAAGTCGCCACGGA[A/T] CTCGGGGCAACAGGCAGATT & 54.5 & 54.5 & 56.5 \\
\hline
\end{tabular}


Table S3 Jointed effects of NRN1 and CAT on NIHL by using the logistic regression models

\begin{tabular}{llllll}
\hline NRN1 & CAT & Risk of NIHL & & \\
\cline { 3 - 6 } rs3805789 & rs7943316 & Controls/Cases, n $(\%)$ & ORs $(95 \%$ CI) & 1.00 & FDR \\
\hline CC & AA & $55(17.9) / 39(12.7)$ & $2.276(1.171-4.427)$ & 0.015 & 0.041 \\
CC & AT & $29(9.4) / 41(13.4)$ & $0.650(0.173-2.442)$ & 0.524 & 0.64 \\
CC & TT & $9(2.9) / 4(1.3)$ & $2.213(1.273-3.849)$ & 0.005 & 0.028 \\
CT & AA & $61(19.9) / 87(28.3)$ & $1.036(0.578-1.857)$ & 0.905 & 0.905 \\
CT & AT & $67(21.8) / 48(15.6)$ & $3.169(1.425-7.048)$ & 0.005 & 0.028 \\
CT & TT & $14(4.6) / 28(9.1)$ & $1.620(0.419-6.205)$ & 0.154 & 0.282 \\
TT & AA & $33(10.7) / 34(11.1)$ & $1.048(0.514-2.137)$ & 0.898 & 0.905 \\
TT & AT & $31(10.1) 23(7.5)$ & $0.558(0.131-2.377)$ & 0.430 & 0.591 \\
TT & TT & $8(2.6) / 3(1.0)$ & $2.005(1.200-3.348)$ & 0.008 & 0.029 \\
CT+TT & AA & $94(30.6) / 121(39.4)$ & $1.892(1.008-3.550)$ & 0.047 & 0.103 \\
CC & AT+TT & $38(12.4) / 45(14.7)$ & $1.255(0.752-2.094)$ & 0.385 & 0.591 \\
CT+TT & AT+TT & $120(39.1) / 102(33.2)$ & & &
\end{tabular}

NRN1: neuritin 1; CAT: catalase; ORs: Odds ratio; CI: confidential interval.

*ORs $(95 \% \mathrm{CI})$ and $\mathrm{P}$ value were obtained with the use of multivariate logistic regression analysis after adjustment for age, gender, education, years of exposure, kurtosis, CNE, adj-CNE, smoking, video volume, physical exercise, and working pressure.

FDR (false discovery rate) was obtained with the use of Benjamin-Hochberg procedure for controlling the false positive rate in multiple comparison. 
Table S4 Jointed effects on NIHL between high-risk genotypes of NRNland CAT and kurtosis

\begin{tabular}{|c|c|c|c|c|c|}
\hline \multicolumn{2}{|c|}{ Combination of kurtosis and genotypes of $N R N l$ and $C A T$} & \multirow{2}{*}{$\frac{\text { Controls/Cases, n (\%) }}{9(2.9) / 8(2.6)}$} & \multirow{2}{*}{$\frac{\text { ORs }(95 \% \mathrm{CI}) *}{1.000}$} & \multirow[t]{2}{*}{$P^{*}$} & \multirow[t]{2}{*}{ FDR } \\
\hline \multirow{12}{*}{ Steady-state noise } & rs3805789 CC- rs7943316 AA & & & & \\
\hline & rs3805789 CC- rs7943316 AT & $6(2.0) / 9(2.9)$ & $2.499(0.561-11.133)$ & 0.230 & 0.814 \\
\hline & rs3805789 CC- rs7943316 TT & $3(1.0) / 0(0.0)$ & 0.000 & 0.999 & 1.000 \\
\hline & rs3805789 CT- rs7943316 AA & $19(6.2) / 14(14.6)$ & $1.317(0.375-4.626)$ & 0.667 & 1.078 \\
\hline & rs3805789 CT- rs7943316 AT & $15(4.9) / 7(2.3)$ & $0.817(0.206-3.234)$ & 0.773 & 0.895 \\
\hline & rs3805789 CT- rs7943316 TT & $3(1.0) / 2(0.7)$ & $0.992(0.122-8.078)$ & 0.994 & 1.047 \\
\hline & rs3805789 TT- rs7943316 AA & $7(2.3) / 5(1.6)$ & $1.289(0.269-6.185)$ & 0.751 & 0.987 \\
\hline & rs3805789 TT- rs7943316 AT & $4(1.3) / 2(0.7)$ & $0.734(0.098-5.485)$ & 0.763 & 0.941 \\
\hline & rs3805789 TT- rs7943316 TT & $1(0.3) / 0(0.0)$ & 0.000 & 1.000 & 1.000 \\
\hline & rs3805789 CT/TT- rs7943316 AA & $26(8.5) / 19(6.2)$ & $1.273(0.388-4.179)$ & 0.691 & 1.013 \\
\hline & rs3805789 CC- rs7943316 (AT+TT) & $9(2.9) / 9(2.9)$ & $1.723(0.422-7.040)$ & 0.449 & 1.041 \\
\hline & rs3805789 (CT+TT)- rs7943316 (AT+TT) & $23(7.5) / 11(3.6)$ & $0.772(0.220-2.710)$ & 0.686 & 1.013 \\
\hline \multirow{10}{*}{ Complex noise } & rs3805789 CC- rs7943316 AA & $46(15.0) / 31(10.1)$ & $1.540(0.348-6.809)$ & 0.569 & 1.043 \\
\hline & rs3805789 CC- rs7943316 AT & $23(7.5) / 32(10.4)$ & $3.347(0.740-15.139)$ & 0.117 & 0.644 \\
\hline & rs3805789 CC- rs7943316 TT & $6(2.0) / 4(1.3)$ & $1.336(0.193-9.262)$ & 0.770 & 0.895 \\
\hline & rs3805789 CT-rs7943316 AA & $42(13.7) / 73(23.8)$ & $3.875(0.895-16.774)$ & 0.070 & 0.051 \\
\hline & rs3805789 CT-rs7943316 AT & $52(16.9) / 41(13.4)$ & $1.689(0.391-7.288)$ & 0.483 & 0.966 \\
\hline & rs3805789 CT-rs7943316 TT & $11(3.6) / 26(8.5)$ & $5.961(1.219-29.155)$ & 0.028 & 0.374 \\
\hline & rs3805789 TT-rs7943316 AA & $26(8.5) / 29(9.4)$ & $2.625(0.581-11.864)$ & 0.210 & 0.843 \\
\hline & rs3805789 TT-rs7943316 AT & $27(8.8) / 21(6.8)$ & $1.750(0.380-8.057)$ & 0.473 & 0.966 \\
\hline & rs3805789 CT+TT-rs7943316 AA & $68(22.1) / 102(33.2)$ & $1.607(1.035-2.494)$ & 0.034 & 0.051 \\
\hline & rs3805789 CC-rs7943316 (AT+TT) & $23(9.4) / 36(11.7)$ & $1.378(0.761-2.495)$ & 0.290 & 0.800 \\
\hline
\end{tabular}


$*$ ORs $(95 \% \mathrm{CI})$ and $P$ value were obtained with multivariate logistic regression analysis after adjustment for age, gender, education, years of exposure, smoking, video volume, physical exercise, and working pressure. FDR (false discovery rate) was obtained from the Benjamin-Hochberg procedure. 


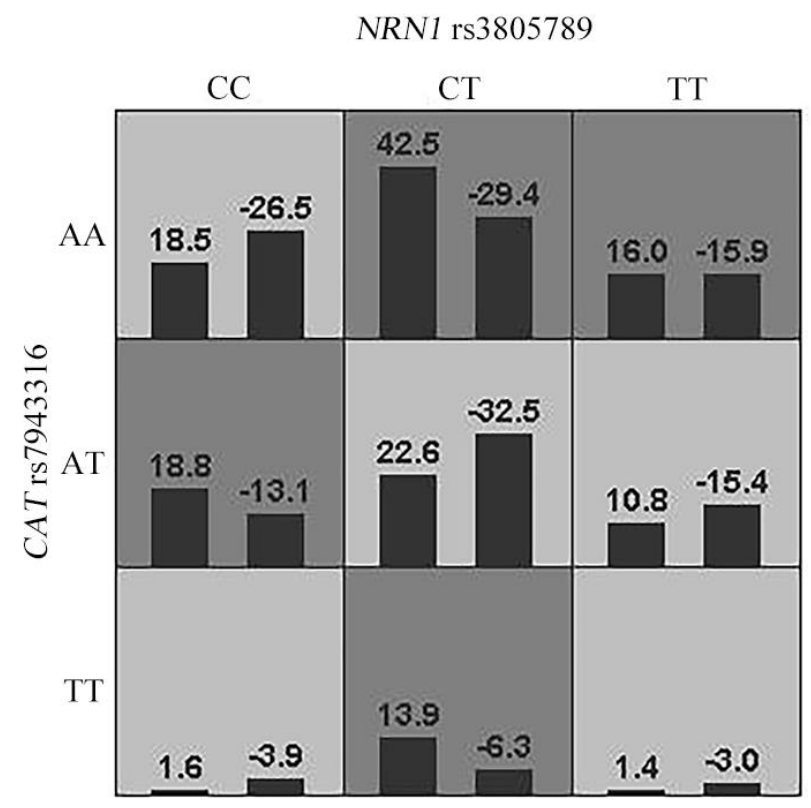

Figure S1 Interactions of NRN1 rs3805789 and CAT rs7943316 associated with NIHL risk identified by GMDR analysis. In each cell, the left bar represents a positive score and the right bar a negative score. High-risk cells were indicated by dark shading, low-risk cells by light shading.

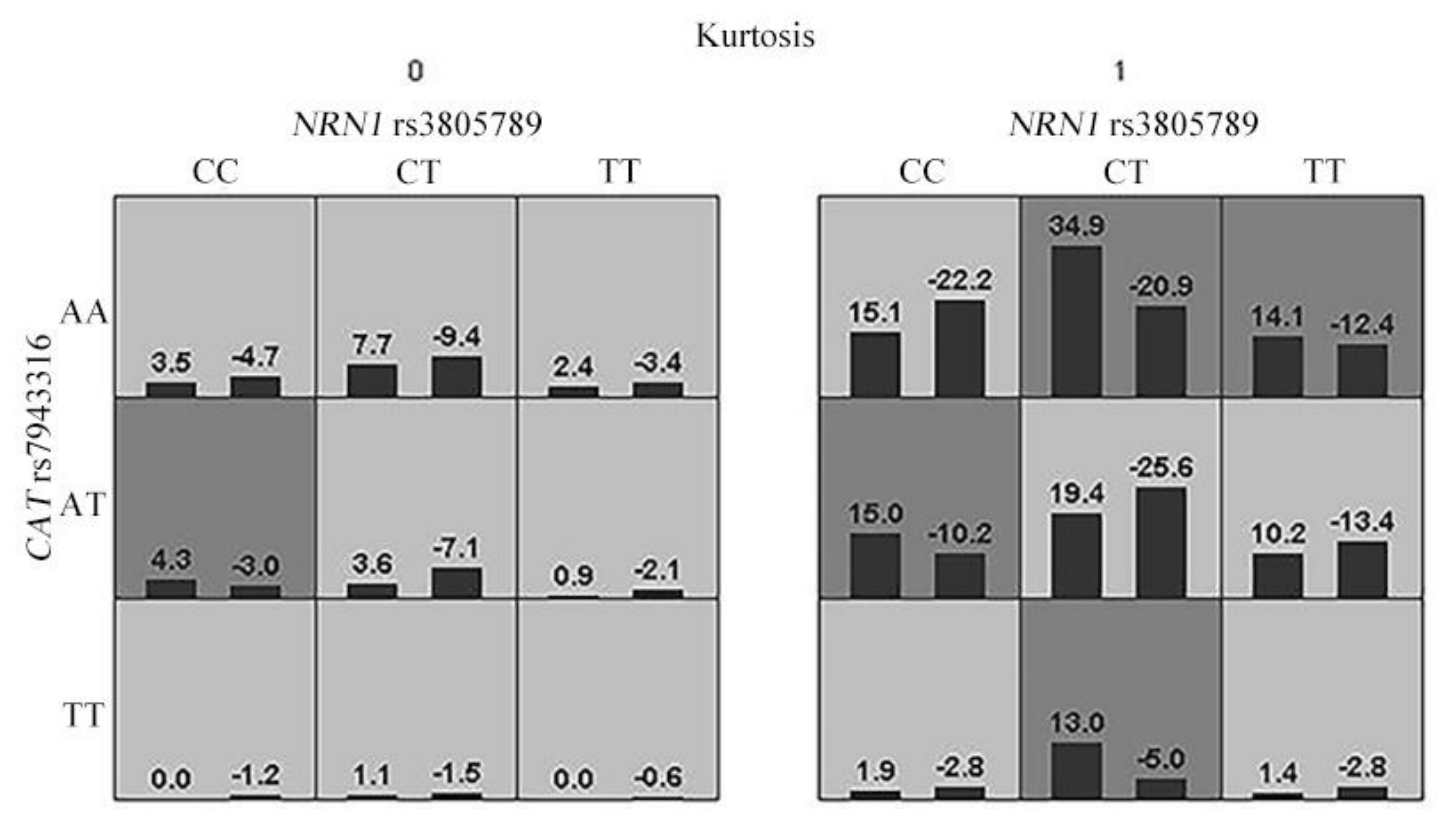

Figure S2 Interactions of NRN1 rs3805789, CAT rs7943316 and kurtosis associated with NIHL risk identified by GMDR. 0: Steady-state noise; 1: Complex noise. In each cell, the left bar represents a positive score and the right bar a negative score. High-risk cells were indicated by dark shading, low-risk cells by light shading. 
Figures

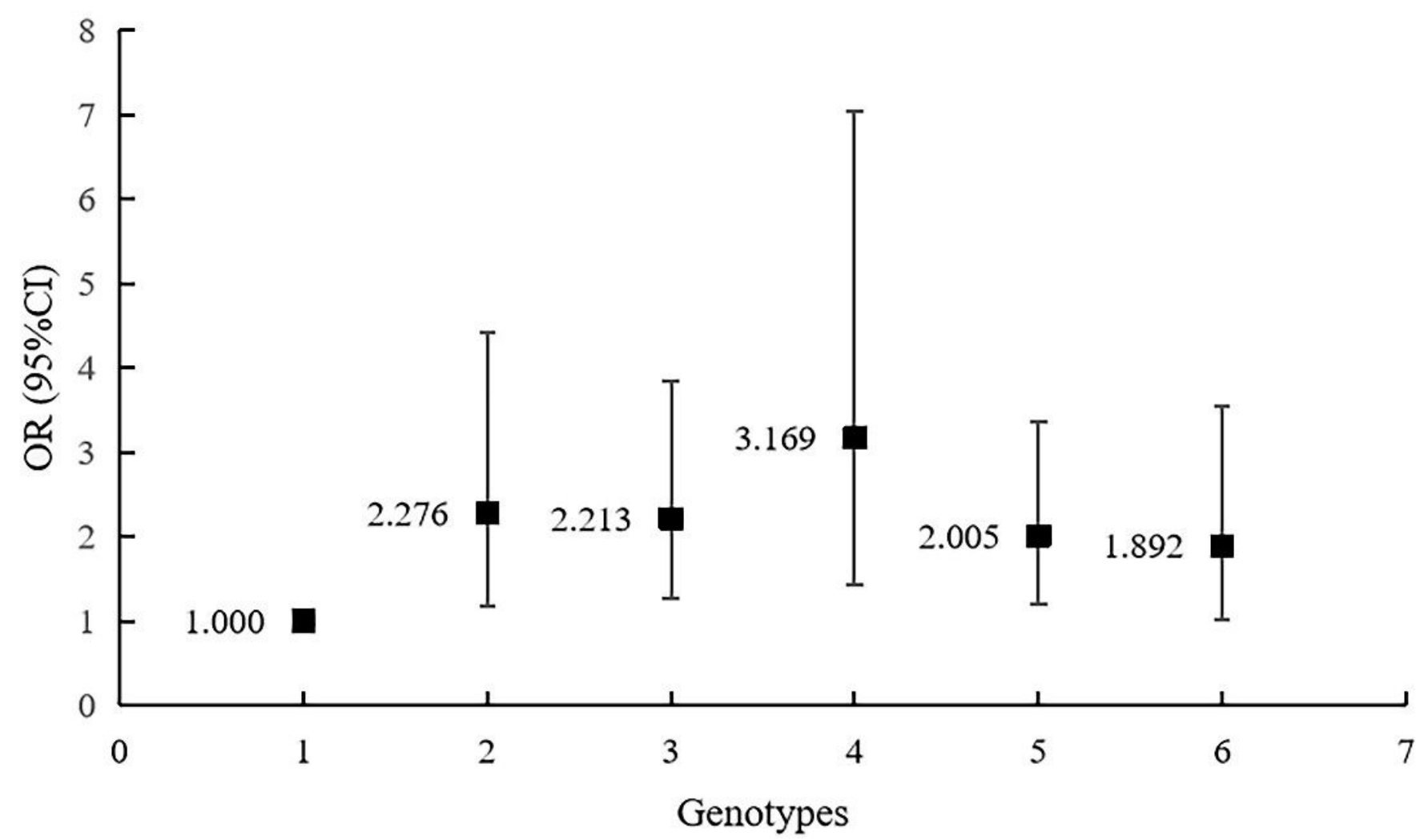

Figure 1

Stratified analysis for gene-gene interaction on $\mathrm{NIHL}$ risk using logistic regression. The odds ratios (ORs) were calculated after adjustment for age, gender, education, years of exposure, kurtosis, CNE, adj-CNE, smoking, video volume, physical exercise, and working pressure. 1: rs3805789-CC and rs7943316-AA; 2: rs3805789-CC and rs7943316-AT; 3: rs3805789-CT and rs7943316-AA; 4: rs3805789-CT and rs7943316TT; 5: rs3805789-CT/TT and rs7943316-AA; 6: rs3805789-CC and rs7943316-AA/TT. 


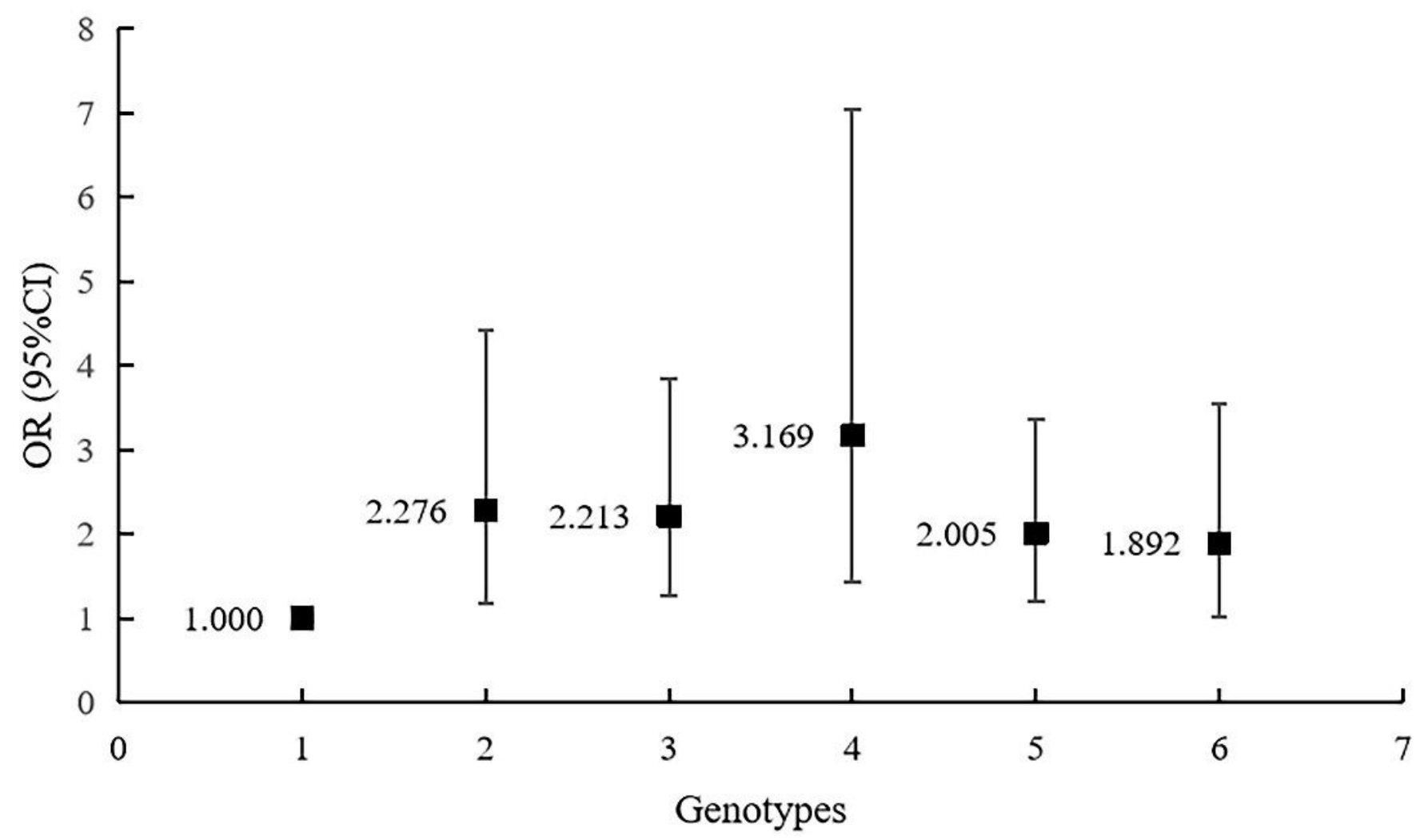

Figure 1

Stratified analysis for gene-gene interaction on $\mathrm{NIHL}$ risk using logistic regression. The odds ratios (ORs) were calculated after adjustment for age, gender, education, years of exposure, kurtosis, CNE, adj-CNE, smoking, video volume, physical exercise, and working pressure. 1: rs3805789-CC and rs7943316-AA; 2: rs3805789-CC and rs7943316-AT; 3: rs3805789-CT and rs7943316-AA; 4: rs3805789-CT and rs7943316TT; 5: rs3805789-CT/TT and rs7943316-AA; 6: rs3805789-CC and rs7943316-AA/TT. 


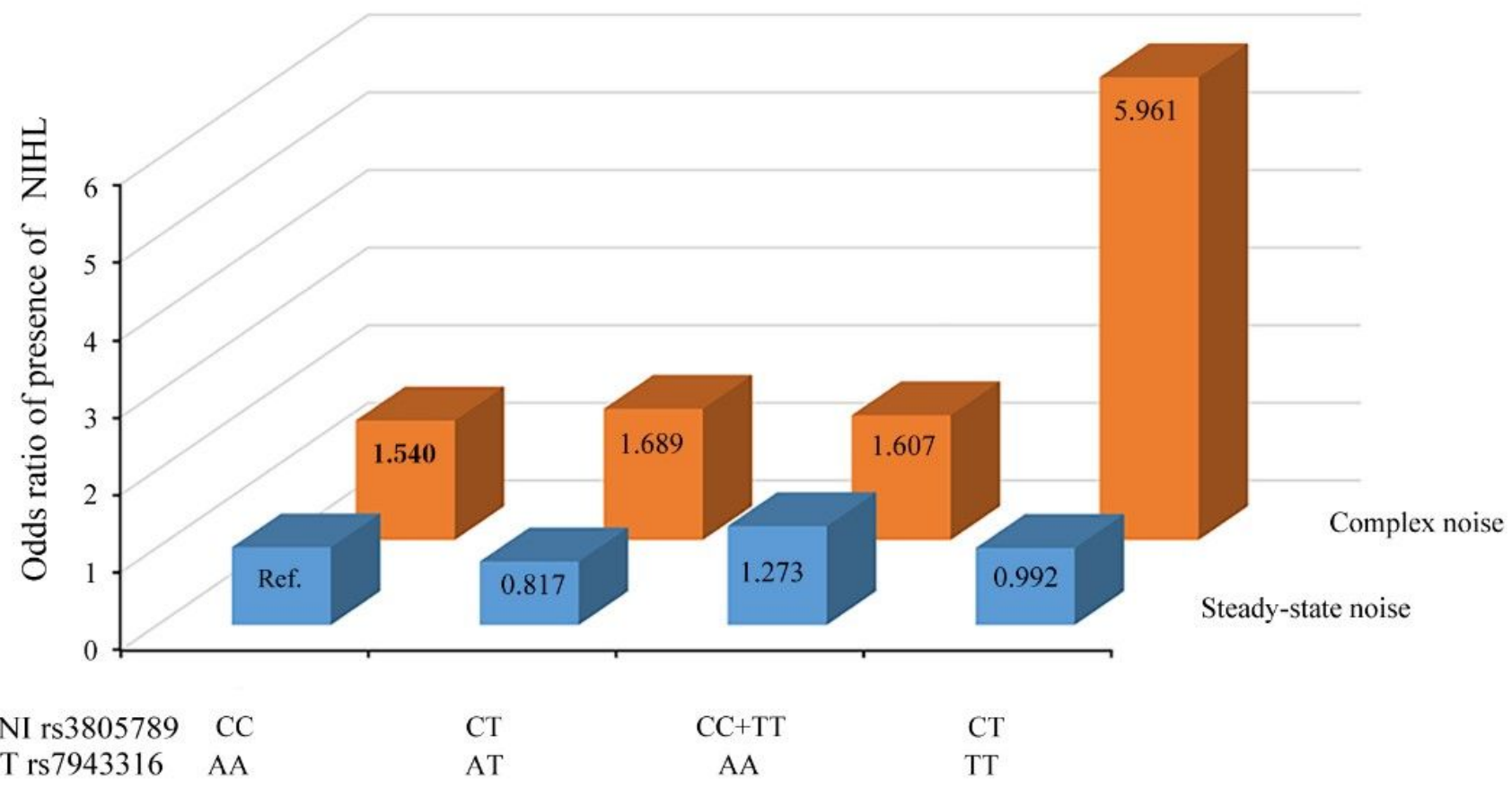

Figure 2

Joint effects of the NRN1 rs3805789 and CAT rs7943316 with kurtosis on NIHL risk. The reference group was defined as subjects exposed steady-state noise who carry NRN1 rs3805789 CC and CAT rs7943316 AA. Ref: reference group. The odds ratios (ORs) were calculated by the logistic regression analysis after adjustment for age, gender, education, years of exposure, smoking, video volume, physical exercise, and working pressure. 


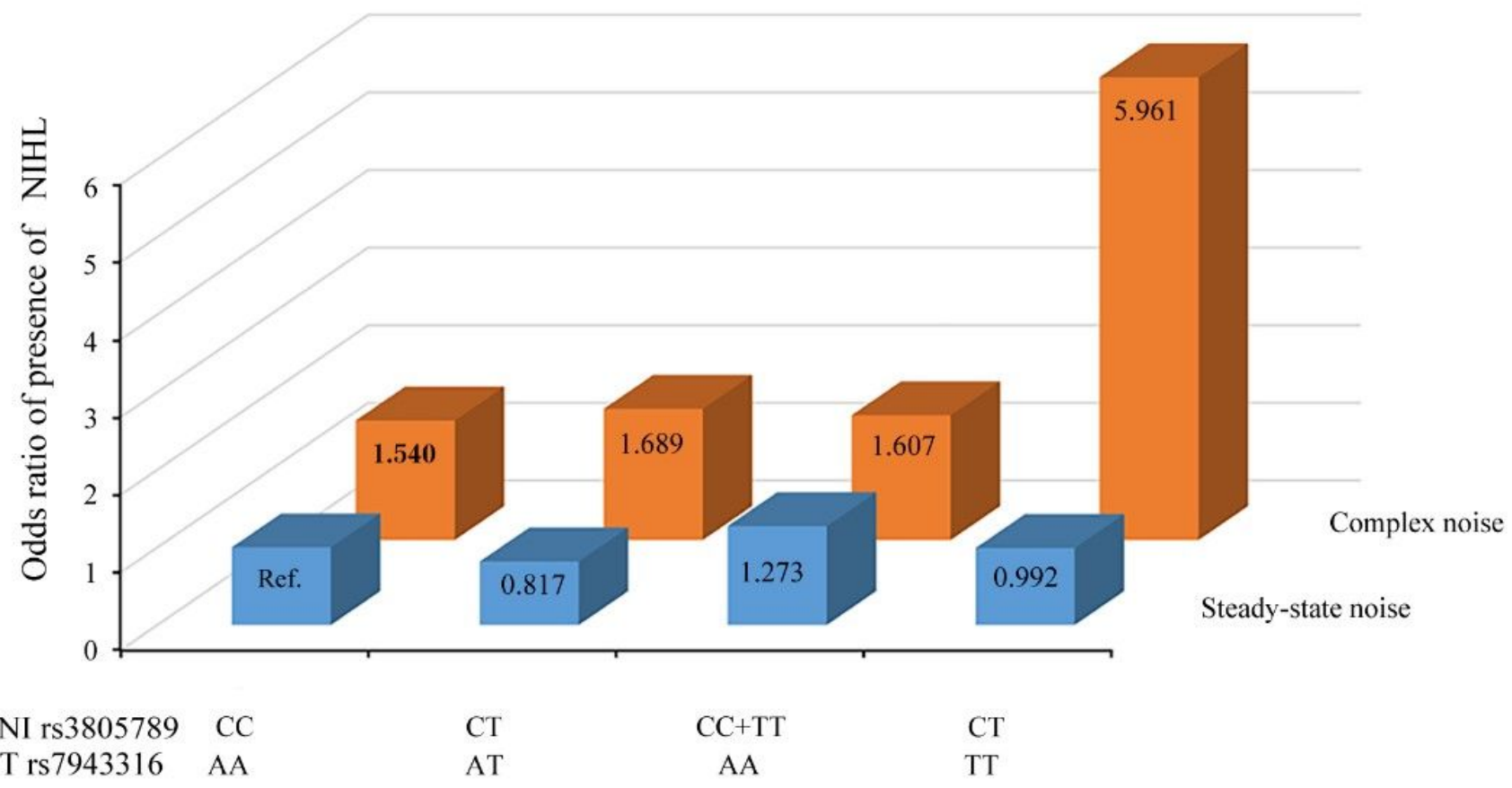

Figure 2

Joint effects of the NRN1 rs3805789 and CAT rs7943316 with kurtosis on NIHL risk. The reference group was defined as subjects exposed steady-state noise who carry NRN1 rs3805789 CC and CAT rs7943316 AA. Ref: reference group. The odds ratios (ORs) were calculated by the logistic regression analysis after adjustment for age, gender, education, years of exposure, smoking, video volume, physical exercise, and working pressure.

\section{Supplementary Files}

This is a list of supplementary files associated with this preprint. Click to download.

- Supplementarymaterial.zip

- Supplementarymaterial.zip 